\title{
Nachhaltige Forstwirtschaft und Holzsparkunst - Frühe Formen des Umgangs mit Ressourcenknappheit
}

Unter dem Eindruck eines von Europa ausgehenden und nunmehr schon über 200 Jahre dauernden spektakulären weltwirtschaftlichen Wachstumsprozesses und dem damit einhergehenden Ressourcenverzehr haben sich namhafte Personen und Institutionen der Frage gestellt, ob noch Jahrhunderte oder gar Jahrtausende »fortschrittlicher Zukunft « vor uns liegen können. Es war vor allem der mit dem Wirken von Dennis L. Meadows verbundene Report über die »Grenzen des Wachstums «, ein Bericht des "Club of Rome " zur Lage der Menschheit, ${ }^{1}$ der bei vielen die Vorstellung eines grenzenlosen materiellen Fortschritts in einer begrenzten Welt zerstörte. Diese Publikation, die sich einer weltweiten Verbreitung und Diskussion erfreute, hat mit ihren Aussagen und Modellannahmen viel Zustimmung und auch Kritik erfahren. Unüberschaubar ist seither die Fülle der Schriften, Kongresse und Konferenzen, der Gremien, Forschungsinstitute und Professionen, der Programme und politischen Bekundungen und meist mühselig zustande gebrachten politischen Handlungsansätze, die einen Kurswechsel in der Ressourcennutzung einfordern. Unfälle bei der atombasierten Energieerzeugung und heftige Widerstände gegen die Lagerung des dabei erzeugten Atommülls treiben die Diskussion an, und in den Massenmedien steht der ungestaltete und ungebremste Wachstumsprozess mit seinen ökologischen Folgen im Kreuzfeuer der Kritik. Im tatsächlichen oder vermeintlichen Bestreben, nicht die Lebensgrundlagen der nachkommenden Generationen zu gefährden, hat ein schillerndes Prinzip der »Nachhaltigkeit « in Wissenschaft und Politik Karriere gemacht.

Vor diesem Hintergrund hat sich die deutsche Geschichtswissenschaft vor allem seit den 1980er Jahren mit der Umwelt-, Ressourcen- und Energieproblematik in der vorindustriellen Zeit, in der Frühen Neuzeit, befasst. ${ }^{2}$ Sie hat dem Bedürfnis nach historischem Orientierungswissen Rechnung getragen und damit erneut bestätigt, dass es zeitgebundene Probleme sind, die in erheblichem Maße den Blick in die Vergangenheit konturieren. Sie hat eine lange vergessene Seite des Wirtschaftens und vergangene Erfahrungen und Diskussionen vergegenwärtigt. Die Geschichtswissenschaft hat historische Wurzeln der aktuellen Probleme offengelegt, Parallelen verzeichnet und die Vergangenheit mit der Gegenwart vermittelt. Sie hat sich durch Gegenwartsprobleme und einen Wandel des Zeitgeistes in der Gegenwart und durch die Umweltbewegung zu einem neuen Analyseschwerpunkt und einem Perspektivwechsel inspirieren lassen. Sie gewährt Einblicke in vergangene »Problemlösungen «

$1 \mathrm{Vgl}$. Meadows et al. 1972.

2 Vgl. als Überblick Brüggemeier 2003; Freytag 2006; Reith 2011.

Leviathan, 42. Jg., 3/2014, S. 433- 462 
und vermittelt historische Erfahrungen. Durchlebte Entwicklungen, Wege und Irrwege werden verdeutlicht. Ein verblüffendes » Wiedererkennen « der Gegenwart nun auch in der Umweltgeschichte wird ermöglicht. ${ }^{3}$ Die Geschichtswissenschaft hat dabei ein Feld historischer Praxen und Diskurse erschlossen, das den heutigen Ansätzen zur Verhinderung einer zukünftigen Ressourcenknappheit durch Ressourceneinsparung und Substitutionsstrategien ähnelt. Erkennbar ist in vielen Beiträgen das Bemühen, aus der Vergangenheit Umrisse eines Modells einer zukunftsfähigen Entwicklung oder Anhaltspunkte eines Vergleichs mit der heutigen Zeit zu gewinnen.

Erstaunlich ist, dass trotz der bereits vorliegenden Ergebnisse der historischen Forschung die allgemeine Diskussion über die Ressourcenknappheit derzeit ohne eine tragfähige Einbeziehung der historischen Dimension geführt wird. Sie ist im Allgemeinen sogar mehr oder weniger geschichtsblind. So erscheint vieles als völlig neu, obwohl namentlich in den vorindustriellen, den agrarischen beziehungsweise frühneuzeitlichen Gesellschaften des 16. bis frühen 19. Jahrhunderts ein weitverzweigter Diskurs über die Ressourcenknappheit in deutschsprachigen historischen Quellen geführt wurde. Auch damals wurden Auswege aus der Ressourcenknappheit diskutiert. ${ }^{4}$

Über die Struktur, die politische Stoßrichtung und den realen Hintergrund dieses Diskurses wird inzwischen in der Geschichtswissenschaft nicht mehr nur gestritten, sondern auch ergebnisbezogen geforscht. ${ }^{5}$ Die Ergebnisse der historischen Forschung zur Ressourcenproblematik sind gut geeignet, die Naturgebundenheit der menschlichen Existenz und des Wirtschaftens zu verdeutlichen und die Geschichte heutiger Problemlagen zu skizzieren. Die Frühe Neuzeit erscheint als Vor- und Ausgangsbedingung des kapitalistischen Industriesystems, des »industriellen Durchbruchs «, als lang andauernder Prozess hin zur » modernen Welt ", in dessen Verlauf die Verfügbarkeit von und der Umgang mit Ressourcen zu einem »Schlüsselproblem « avancierte, ${ }^{6}$ das zwar auf den ersten Blick gelöst wurde, aber ohne Rücksicht auf die Nachhaltigkeit. Das hat auf den zweiten Blick mehr Kosten als Nutzen gebracht.

\section{Der Ressourcendiskurs in der deutschen Geschichte}

\subsection{Holz als zentrale und bedrohte Ressource der Agrargesellschaft}

Blickt man von der "Höhe der Jetztzeit « auf die vorvergangenen Zeiten, gilt es eine spezifische "Fremdheit " der hier behandelten Jahrhunderte seit dem Ausgang des Mittelalters zu beachten. Dass es sich im deutschen Sprachraum und weit darüber hinaus um eine überwiegend ständisch-feudal strukturierte Agrargesellschaft gehandelt hat, kann und soll diese Andersartigkeit nur andeuten. Hinzu tritt eine große

\footnotetext{
3 Abelshauser 1994, S. 7 ff.

4 Gleitsmann 1981, S. 33 ff.

5 Vgl. Hölzl 2010.

6 Dietz 1997, S. 143 ff.
} 
Vielgestaltigkeit und ein enormer sozialer, ökonomischer und politischer » Wandel « in einem Zeitraum, an dessen Beginn - je nach der eingenommenen Perspektive die italienische Renaissance, der Humanismus, die Entdeckung der »Neuen Welt", die Reformation oder der Bauernkrieg stand und dessen Ende durch die amerikanische Verfassung, die Französische Revolution, den Zusammenbruch des Heiligen Römischen Reichs und den Durchbruch der »industriellen Revolution « geprägt war. $^{7}$

Die Zentralressource der damaligen Zeit war bis in das 19. Jahrhundert hinein das Holz. Es war unersetzlicher Bau-, Brenn- und Werkstoff, es war Fundament des Gewerbes, des Handwerks und Handels, der vormodernen gesellschaftlichen Ordnung und Macht. Es war darüber hinaus Ausgangsstoff für die Herstellung von Holzkohle, Pottasche, Pech und Teer. Eichen lieferten die begehrte Lohrinde für das Gerben, Kiefernharz war der Grundstoff für die Terpentinherstellung. Holz war in den »kälteren Gegenden « und während der langen Jahre der »Kleinen Eiszeit" zudem die wichtigste Voraussetzung und Grundlage für das Überleben der Menschen überhaupt. Der Wald, sein Zustand und seine Pflege waren von höchster Bedeutung. Er war darüber hinaus ein Ort vielfältiger Nutzungen, forstwirtschaftlicher Überlegungen und hoheitlicher Regulierungen ${ }^{8}$ und entsprechender Interessen. Wald und Holz standen im Mittelpunkt des Diskurses um Ressourcenknappheit. Dieser drehte sich schwerpunktmäßig um Fragen der »optimalen « Holzgewinnung, der Holznutzung, der Holznot, der Holzeinsparung und der Holzsubstitution. Vor diesem Hintergrund sprach Sombart ${ }^{9}$ von einem »hölzernen Gepräge « der vorindustriellen Zeit beziehungsweise des »Frühkapitalismus «. Es war die Zeit einer bis ins 19. Jahrhundert reichenden, im Wechselspiel politischer, ökonomischer und technologischer Veränderungen zunehmend »zerfallenden «, überwiegend ständisch-agrarisch geprägten Wirtschafts-, Herrschafts- und Gesellschaftsordnung. ${ }^{10}$

Angesichts der vergleichsweise noch geringen Bedeutung von Metallen und von anderen Bau-, Werk- und Brennstoffen ist die Begriffsbildung Sombarts eine Pointierung, die man durchaus nachvollziehen kann. Moderne Zentralressourcen beziehungsweise Energieträger wie Kohle, Gas und Erdöl wurden nur wenig oder sehr spezifisch genutzt. Ein regelrechtes "Zeitalter « der Kohle und der Kohlenwasserstoffe begann erst mit der Industrialisierung und der Entwicklung der Eisenbahn im 19. Jahrhundert.

Bei der vor allem nach 1945 herbeigeforschten, herbeigesehnten, aber auch kritisierten und schließlich gebauten und produzierten Atomenergie handelt es sich um eine Technologie, die erst durch die physikalischen Theorien des 20. Jahrhunderts ermöglicht wurde und die in den hier untersuchten Zeiten gar nicht erkannt geschweige denn umfassend genutzt werden konnte. Auch von einer kommerziellen Erzeugung und Nutzung der Elektrizität konnte keine Rede sein.

7 Reith 2011, S. 5.

8 Vgl. Ernst 2000.

9 Sombart 1987 [1916], Bd. 2, S. 1137 ff.

10 Vgl. Volckart 1998.

Leviathan, 42. Jg., 3/2014 
Die in der Geschichtswissenschaft geführte Großkontroverse um das reale Ausmaß und die Gestalt der Holznot ${ }^{11}$ hat ergeben, dass von einer allgemeinen Ressourcenknappheit in der hier zur Diskussion stehenden Zeit nicht auszugehen ist. Es habe aber lokal und regional abgrenzbare, zeitlich sowie sozial umreißbare Knappheits- und Krisenerfahrungen gegeben. Mangelsituationen konnten damals in vielen Fällen durch Transporte nicht oder nicht ausreichend ausgeglichen werden. Die Ressourcenknappheit breitete sich vor allem im Umfeld von großen Städten, von Transportwegen und vorindustriellen Produktionsstandorten aus. ${ }^{12}$ Die Ressourcenknappheit folgte auch einem zeitlichen Muster. Es scheint besonders im 18. Jahrhundert einen verbreiteten Trend zur Knappheit gegeben zu haben. Darin äußerte sich die »menschliche « Tendenz, auch das Holz »[...] in seinen drei Verwendungsarten als Brenn-, Bau- und Werkstoff bis an die äußersten Grenzen der jeweils vor Ort verfügbaren Kapazitäten « auszuschöpfen und »wüste Zustände« zu hinterlassen. ${ }^{13}$ In einer Gesellschaft sozialer Ungleichheit existierte darüber hinaus in jenen sozialen Schichten und Gruppen eine häufig extreme Ressourcenknappheit, in denen den Menschen die Tauschmittel oder Zugangsberechtigungen und Zugänge zum Holz und zu anderen Waldressourcen fehlten beziehungsweise diese gewaltsam verwehrt wurden. ${ }^{14}$

Vor diesem Hintergrund oszilliert der in der Frühen Neuzeit vielbeklagte »Holzmangel « zwischen einem diskursiven und wirklichen Problem. ${ }^{15}$ Neben diesen notwendigen Differenzierungen ist der mitunter deutlich sichtbare interessenpolitische Hintergrund in der historischen Debatte zu berücksichtigen. Festzuhalten bleibt, dass insgesamt in den Beiträgen zur vormodernen Debatte um Ressourcenknappheit ein klares Bewusstsein von der Bedeutung der Zentralressource Holz für die menschliche Kultur existierte, dass häufig bedrohliche Folgen der Holznot angenommen wurden, dass aus allerdings unterschiedlichen Motiven Einigkeit über das Ziel bestand, Holznot zu verhindern, und dass aus den frühneuzeitlichen Quellen eine große Angst spricht, dass der Mensch in der Lage sein könnte, sich seine Lebensgrundlagen zu zerstören. ${ }^{16}$

11 Vgl. zu dieser Kontroverse Radkau 1986; Strittmatter 1986; Radkau, Schäfer 1987, S. 149 ff.; Schäfer 1992, S. 15 ff.

12 Vgl. Dietz 1997.

13 Ebd., S. 151. Auf der Basis erweiterungsbedürftiger Quellen unterscheidet Gleitsmann bis ins 19. Jahrhundert hinein sechs Phasen der Waldnutzung seit dem Mittelalter, die jeweils Tendenzen zur Ruinierung oder zur Erholung der Wälder beinhalteten. Erholungsphasen der Wälder sieht er vor allem in Abhängigkeit von demographischen Faktoren. Sollten sich diese Zusammenhänge erhärten lassen, wären sie ein Menetekel (Gleitsmann 1989, S. 183 ff.).

14 Vgl. Radkau 1999.

15 Ebd., S. 102; Grewe 2004 a, S. 38.

16 Vgl. Radkau 1986. 


\subsection{Holznot und Waldverwüstung}

Das Montangewerbe, eines der ältesten Gewerbe der Menschheit, war schon vor Beginn der Frühen Neuzeit immer wieder geplagt von der Angst vor einer bedrohlichen Holznot. ${ }^{17}$ Das sich stellenweise stark entwickelnde Montanwesen wurde wegen der damit einhergehenden Umweltschädigungen bereits gegen Ende des 15 . Jahrhunderts als Schändung der "Mutter Natur «, als »verwüstende Tat « kritisiert, und der Bergbau treibende Mensch erschien als »Mörder der Natur «. Der auch zu jener Zeit unwiederbringlich ausbeutend voranschreitende und seinem Wesen nach nicht nachhaltige Bergbau, das Graben nach den "Schätzen der Erde ", war auf den ergänzenden Einsatz der regenerativen Ressource Holz für die Grubenzimmerung angewiesen. ${ }^{18}$ Der Bergbau war immer schon Gegenstand hoheitlicher Regulierungen, und er beanspruchte den Wald nicht selten über die Grenzen seiner Regenerationsfähigkeit hinaus. Hinzu traten über Jahrhunderte hinweg weitere vorindustrielle Gewerbe (zum Beispiel Salinen, Köhlereien, metall- und glaserzeugende Fabrikationsstätten), die im lokalen und regionalen Maßstab wahre »Waldfresser" und »Waldverwüster « waren. Trotzdem wurden diesen Gewerben ganze Waldungen »gewidmet «. Sie hatten in solchen Fällen Anrecht auf gewaltige Holzmengen zu niedrigen Preisen. Auch vorindustrielle Maschinen, Bauwerke, viele Gebrauchsgegenstände, die Kriegsflotten, Schiffe, Boote und Wagen - also beinahe alles - vernutzte ein bedrohtes »Stück Wald «. ${ }^{19}$

Es existierte ein breites Panorama von Anpassungen des Gewerbes an die Ressourcenknappheit. Auch für die weitreichende Nutzung von Naturkräften (Wasser, Wind) kannte man solche Anpassungen in Form von Gewerbebeschränkungen der verschiedensten Art. Aber es lassen sich auch Strategien des »Überspielens « lokal oder regional auftretender Holznot erkennen, durch Importe, insbesondere wo es Transportmöglichkeiten aus auswärtigen waldreichen Gegenden gab und wo entsprechende Handelsbeziehungen das ermöglichten. Ansonsten galten die natürlichen Stoffe und Kräfte in der Agrargesellschaft als »unhintergehbare " Engpassfaktoren, die das Produktionsniveau in der vorindustriellen Gesellschaft begrenzten. Das geschah allerdings auch durch »Krisen alten Typs « wie zum Beispiel Naturund Hungerkatastrophen, Seuchen, Kriege und verschiedenartige Störungen des Handels.

Eindrucksvoll ist das in den zeitgenössischen Quellen entfaltete Panorama der Schädigungen und Gefährdungen des Holzes, dieser knappen, biologischen und eben deshalb regenerativen Zentralressource der vorindustriellen Gesellschaft: Die Eichel- und Buchenmast, der Verbiss durch Haus- und Wildtiere, der Holzexport, Sturmschäden, Dürre und Sommerhitze, Waldbrände, Baumkrankheiten, tierische Holzschädlinge insbesondere in den schon damals angelegten Holzplantagen, Fehl-

17 Vgl. Lohrmann 1979.

18 Vgl. zu den Sprachbildern die allegorische Erzählung des sächsischen Humanisten Paulus Niavis (Paul Schneevogel) aus dem Jahre 1492: Bartels 2000, S. 41 f.; weitere Hinweise bei Radkau 1986, S. 71 f.

19 Gleitsmann 1981, S. 39 ff.

Leviathan, 42. Jg., 3/2014 
verhaltensweisen der Forstbeamten und »Bedienten " sowie der »Unterthanen ", zahlreiche Fehler bei der Ernte des Holzes, beim »Hartz-Reissen «, bei der Ernte der Waldfrüchte, bei der Pflege und Aufforstung trugen nach der Auffassung der Holznotautoren und der forstwirtschaftlichen Experten zum Niedergang der Wälder und des nutzbaren Holzes bei. Hinzu kam noch die Ausdehnung des Acker-, Teich-, Feld-, Wiesen- und Gartenbaus, die zu Lasten des Waldes ging. Eine nicht untypische Sicht der Ressourcenknappheit formulierte der Verfasser des Stichworts » Wald « in Zedlers Universallexikon: Früher sei der größte Teil Deutschlands eine »Waldung « gewesen, und es sei deshalb Holz in großer Menge vorhanden gewesen. Das Holz habe man darum wenig geachtet. Bergwerke habe es nur wenige gegeben, »[...] viel Künste, Profeßionen und Handwercke, die theils der Fleiß, theils die Thorheit ausfündig gemacht, waren dahmals unbekannt, bey denen in den jetzigen Zeiten eine unsägliche Menge des Holtzes verthan wird. Bey dem Kochen gieng in der damahligen alten Welt auch nicht so viel drauf, denn unsere alten Deutschen hielten viel auf kalte Küche $[. .$.$] «. { }^{20}$ »Zeithero « habe das »Holtzhauen « überhandgenommen. Eingerahmt in die Schilderung der schrecklichen Folgen der Holznot zitiert der Verfasser den »seligen « Luther, der geweissagt habe, »[...] es werde Deutschland vor dem jüngsten Tage an drey nöthigen Eigenschafften mangeln, [...] an guten aufrichtigen Freunden, an tüchtiger und wichtiger Müntze, und an wilden Holtze, welches leider! die tägliche Erfahrung mehr als gar zu gewiß bezeuget «. ${ }^{21}$

In diesem Zusammenhang stand die Forderung nach einer ebenso effektiven wie »nachhaltigen « Bewirtschaftung des Waldes, um nicht durch Raubbau an der Zentralressource Holz kurzfristig zwar Gewinn zu machen, langfristig aber Mangel, Elend, Not und Tod heraufzubeschwören. Als "Erfinder « und Vertreter der Forderung nach einer solchen nachhaltigen Nutzung der Zentralressource Holz gilt heute der Oberberghauptmann Hannß Carl von Carlowitz, einer der einflussreichsten Männer im Sachsen August des Starken, mit seiner Schrift Sylvicultura oeconomica. ${ }^{22}$ Der Sache nach war schon damals die Idee der nachhaltigen Ressourcennutzung mit den verschiedensten Interessen verbunden, entsprechend vieldeutig, wandelbar, uralt und global verbreitet. ${ }^{23}$

So kann es nicht überraschen, dass sich Spuren des Nachhaltigkeitsdenkens auch im älteren Forstrecht, in den Forstordnungen finden, die in diesem Sinne die Nutzungsberechtigungen und -verbote der am Holz, an der Landwirtschaft im Wald und an der Jagd interessierten Kräfte regulieren sollten. Sie spiegelten zudem das Bemühen, nach Kriegen und anderen Katastrophen überhaupt eine Ordnung in den Wäldern wiederherzustellen, und fügten sich in die allgemeine Tendenz der Frühen

20 Zedler 1747, Sp. 1160.

21 Ebd., Sp. 1161.

22 Vgl. von Carlowitz 1713.

23 Grober 2001, S. 4; Karafyllis 2002. 
Neuzeit zu einer zunehmenden Verrechtlichung zahlreicher Lebensbereiche. ${ }^{24}$ Als Spiegelbild der politischen Zersplitterung und der historischen Entwicklung existierte eine ungeheure Vielfalt solcher Rechtsquellen mit jeweils ähnlichen Inhalten. ${ }^{25}$ Die entsprechenden Wald-, Forst-, Holz- beziehungsweise Polizeiordnungen und die dazu publizierten Texte zeichneten typischerweise direkt oder indirekt ein schlimmes Bild damaliger Waldzustände. In diesem Zusammenhang spielten die Hinweise auf einen bereits eingetretenen oder einen zu erwartenden Holzmangel eine zentrale Rolle. Insgesamt sollten sie als waldschädigend qualifizierte Nutzungsformen und vor allem »Forstdevastationen " ausschließen. Zu diesem Zweck enthielten sie Verbote, Strafen und administrative Regelungen. ${ }^{26}$ Auch die »Nachkommen «, deren Holzversorgung ja gesichert werden müsse, gerieten in den Blick. Dabei ist allerdings zu beachten, dass von den Rechtsquellen nicht unmittelbar auf real existierende Waldzustände geschlossen werden darf und frühneuzeitliche Regelungen häufig unter einer spezifischen Geltungs- und Durchsetzungsschwäche litten. ${ }^{27}$ Schließlich war auch die im 16. und 17. Jahrhundert erscheinende Hausväterliteratur vom Schreckbild der Holzverschwendung und Waldverwüstung durchdrungen und redete einer systematischen und am Leitbild der Nachhaltigkeit orientierten Forstpflege das Wort. ${ }^{28}$ Während manche Publikation zu den schlechten Waldzuständen und zur Holznot den Blick auf die Folgen für die »Herrschaft « und die »fürstliche Cammer" und den Wald als rational zu bewirtschaftenden Naturraum richtete, gelang es von Carlowitz, aus der Interessenperspektive des regierenden Fürsten, der Gewerbe und der »armen Unterthanen « zu argumentieren. Sie alle hätten durch den Holzmangel zu leiden. Nur durch eine ausreichende und »immerwährende Holtz-Nutzung " lasse sich auch das (Montan-) Gewerbe erhalten und ausdehnen. Zugleich sei Holz zur »Hinbringung des menschlichen Lebens « und zur Unterhaltung des »allgemeinen Besten« unverzichtbar. Alles hänge am Holz, das »liebe Brodt", Salz und Schmalz, Kochen und Brauen, das Wohnen im Trocknen, die Gesundheit und das Leben vor allem im Winter, die zu Privat- und Staatsreichtum führenden Gewerbe, kurz: die »Landes-Wohlfarth «. So wurde von ihm das Holz als »requisitum primum " erkannt, und er schlussfolgerte, »[...] daß ohne das Holtz und dessen nötig- und nützlichen Gebrauch / das menschliche Leben und Bonum publicum nicht wohl bestehen / noch unterhalten werden könne «. ${ }^{29}$

24 Zahlreiche Hinweise finden sich in den Lexika der damaligen Zeit, etwa bei Zedler 1747. Als Zufallsfund vgl. auch den Titel 20 (Von Holzverwustung und wie dem zuvorkommen sey) des Entwurfs einer Polizeiordnung für das Herzogtum Sachsen-Lauenburg aus dem Jahre 1591 (Hempel 1980, S. 119 ff.).

25 Vgl. die Aufzählung bei Meyer 1851, S. 70 ff.

26 Vgl. die allerdings regional begrenzten Hinweise bei Ernst 2000.

27 Vgl. ebd.

28 Bader; Dilcher 1999, S. 217 ff.

29 So von Carlowitz in dem unpaginierten »Vorbericht « seiner Schrift von 1713.

Leviathan, 42. Jg., 3/2014 


\section{Wege aus der Holznot der Frühen Neuzeit}

\subsection{Nachhaltige Forstwirtschaft und allgemeine nachfragebezogene Strategien}

Mit der in den Forstordnungen skizzierten geregelten Forstwirtschaft, mit dem rationalen Holzanbau, der Waldpflege und einer entsprechend überwachten Reglementierung der Wald- und Holznutzung wurde ein "Ausweg " aus der Holznot beschritten. Bereits im Lichte des älteren Forstrechts erscheint die hohe Zahl der auf dem Wald typischerweise »liegenden " Nebennutzungsrechte, beispielsweise das Mastungsrecht, die Eichellese, das Recht auf Reisigholz, das Waldweide- und Hutrecht, das Waldstreurecht, das Recht auf Harzgewinnung, das Recht auf Gewinnung anderer Rohstoffe im Wald, als hinderlich für eine Walderholung und für einen »nachhaltigen " Holzanbau. Die wenig besiedelten und durch die vormodernen Nutzungsformen geprägten Fluren und die »wilde Natur «, das »Unland «, erschienen den »Fortschrittsmännern" der damaligen Zeit als »öde Strecken", als »mißhandelte Waldungen « und » unübersehbare Moraste «, die durch »Landeskultur « in »blühende Fluren « umzuwandeln seien..$^{30}$ Ein zu hoher Wildbestand wurde kritisiert. Auch manche rechtlich abgesicherte Formen der unentgeltlichen Holzentnahme zur "Nothdurft ", wie das Reisigsammeln, gerieten in die Kritik. Beanstandet wurden ebenso die Formen des Gemeineigentums, der Allmenden, die von ihren Gegnern als »Pflanz-Gärten des Müßigganges « bezeichnet wurden. ${ }^{31}$ Aus alledem erwuchs die Absicht und Praxis der Vertreter der nachhaltigen Forstwirtschaft, die auf dem Wald ruhenden Nebennutzungsrechte abzulösen und sie so aus den Waldungen zu entfernen. ${ }^{32}$ Die Allmenden sollten aufgeteilt und zu Ungunsten der Armen privatisiert, das heißt in Einzeleigentum überführt, Land- und Forstwirtschaft stärker getrennt werden. Um zu einer geregelten Forstwirtschaft zu kommen, sollte die aus der Sicht der Forstwirte als »unvernünftig « geltende agrarische Nutzung der Wälder eingeschränkt oder beendet werden. Die Dorfbevölkerung sollte aus dem Wald und das Vieh in den Stall gedrängt werden. ${ }^{33}$ Die Forderung nach einem Ausbau der Landverkehrsinfrastruktur zur Erleichterung des Holztransports, der Import von Holz und Holzprodukten bei gleichzeitigem Exportverbot sind ebenfalls in diesem Zusammenhang zu sehen.

Diese akute Problematik führte zu einem Neuansatz in der Forstwissenschaft. Es wurde eine spezifische Theorie und Praxis einer »nachhaltigen « Holzwirtschaft entwickelt. Der Planungshorizont für die Waldungen wurde weit in die Zukunft geschoben, und es entstand das Idealbild eines durch Aufforstung und Forstpflege heranzuziehenden und vorzugsweise in Schläge aufgeteilten Hochwaldes. Er sollte zuverlässig und langfristig einen maximalen Ertrag garantieren. Dem Holzmangelargument kam in diesem Zusammenhang eine legitimierende Bedeutung zu. Es gehörte zu einem »ökologischen Metanarrativ«, das tiefgreifende Eingriffe nahe-

30 Beck 2003, S. 189.

31 Holenstein et al. 2007, S. 28.

32 Meyer 1851, S. 90 ff.

33 Gleitsmann 1989, S. 204. 
legte und das die Rettung vor dem Schreckensbild einer allgemeinen Holznot versprach. ${ }^{34}$

Die seit dem Beginn des 19. Jahrhunderts konflikthaft und vielgestaltig in Gang kommende Umgestaltung der Wälder zu »Holzäckern «, zu »Holzplantagen « nahm nun keine Rücksicht mehr auf die Bedürfnisse der bisherigen Formen der Landwirtschaft und auf die »Logik vorindustrieller dörflicher Ökonomien «. ${ }^{35}$ Vor allem wurden die Überlebensinteressen der landarmen und landlosen Bevölkerungskreise nicht mehr angemessen berücksichtigt. Umso mehr wurden die Belange staatlicher Instanzen und die Gewinninteressen privater Waldeigentümer im Zuge dieser Intensivierung der Naturnutzung bedient. ${ }^{36}$

Diese Diskurse und Bestrebungen verwiesen bereits auf die angebliche Notwendigkeit der Überführung der traditionellen Eigentumsformen und Nutzungsberechtigungen am Wald in ein »volles « und »ungestörtes « (Privat-)Eigentum. Im Lichte der sich im späten 18. Jahrhundert verbreitenden liberalen Wirtschaftsdoktrin wurde das Privateigentum zu der Form, »[...] in der sich die >Freiheit und die effektivere, die kompetitive Nutzung der natürlichen Ressourcen realisieren sollte «. ${ }^{37}$ Dieser sich in Preußen in den Reformedikten von 1807 und 1811 niederschlagende Gedanke ${ }^{38}$ führte mittelfristig zu einer grundlegenden Transformation der land- und forstwirtschaftlichen Verhältnisse. Der vielgestaltige Transformationsprozess, der auch die Gewerbeverhältnisse betraf, verursachte eine breite Spur des Forstfrevels aus Gewohnheit und Existenznot. ${ }^{39}$ Die von der Nutzung nunmehr Ausgeschlossenen trafen auf "versperrte ", privatisierte Waldungen. Sie hatten darüber hinaus mit der Tatsache zu leben, dass mit dem forstwirtschaftlich angestrebten höheren Holzertrag erst für die ferne Zukunft zu rechnen war und dass sich das lebenswichtige Brennholz verknappte. Es entwickelte sich ein Holzmarkt, auf dem die Staats- und Privateigentümer als Anbieter auftraten und typischerweise das Ziel der Gewinnmaximierung verfolgten. Ein in der Mitte des 18. Jahrhunderts einsetzendes starkes Bevölkerungswachstum sorgte auf dem Holzmarkt für eine starke Nachfrage und hohe Preise. Die dadurch gesteigerte Existenznot der armen und verarmenden Schichten trug auch zum Feldfrevel bei, der eine Landwirtschaft traf, die zudem wegen des Wegfalls »ihrer « Möglichkeiten, den Waldboden, Laub und andere Pflanzenabfälle als Dünger zu nutzen, in besonderer Weise mit dem Problem der Aufrechterhaltung der Bodenfruchtbarkeit konfrontiert war. Von den "gesetzgebenden Faktoren " aus frühliberaler Gesinnung als äußerst vorteilhaft angepriesen, führte dieser Schritt in den modernen Kapitalismus, in die Kommerzialisierung der ländlichen Verhältnisse, bei seinen Kritikern zu der allerdings falschen Befürchtung,

34 Hölzl 2010, S. 37 ff.

35 Ebd. S. $379 \mathrm{ff}$.

36 Zusammenfassend vgl. ebd., S. $489 \mathrm{ff}$.

37 Beck 2003, S. 153.

38 Hasel 1974, S. 22 ff.

39 Vgl. Prass 1996; Mooser 1984; insgesamt: Koselleck 1989, S. 487 ff.; Grewe 2004 b, S. 195 ff.; Hölzl 2010, S. 267 ff. 
nun werde es aus Habgier oder Geldnot erst recht zu einer großflächigen Zerstörung der Wälder kommen.

Nur noch schwer nachvollziehbar erscheinen aus heutiger Sicht andere Maßnahmen zur Bekämpfung der Holznot. Dazu gehörte die Verpflichtung der »Unterthanen « zur Anpflanzung von Bäumen als Voraussetzung der Trauung und die teilweise Entlohnung mit Holz. In diesen Zusammenhang gehört auch ein preußischer königlicher Spezialbefehl aus dem Jahre 1801. Dieser sollte die »Kirchen- und SchulBedienten " motivieren, zwecks größerer Holz- und Geldersparnis Weichholz als Brennholz auf den »Dienstländereyen " heranzuzüchten. ${ }^{40}$ Beide Maßnahmen atmen noch ganz den Geist der alten, der agrarisch-vorliberalen Zeit.

In der vorindustriellen Gesellschaft wurden weitere Strategien erwogen, um der vieldiskutierten Ressourcenknappheit zu entgehen. Es handelte sich einmal um Überlegungen und praktische Ansätze, durch die verstärkte Nutzung von Torf, Braun- oder Steinkohle den Holzverbrauch einzudämmen, ${ }^{41}$ das heißt auf die »unterirdischen Wälder « zurückzugreifen, auf die »Grabmale « der älteren und ältesten Vegetation. Mit dem Holzmangelargument wurde also ausdrücklich zu einer irreversiblen Ausbeutung der Natur geraten, zum Übergang auf »fossile Brennstoffe «. 42

Tatsächlich begann in Deutschland im 18. Jahrhundert eine Politik zur Substitution der erneuerbaren Zentralressource Holz durch die nicht erneuerbare Kohle als Energieträger. Es waren allerdings weniger die staatlichen Anreize und Interventionen, die diesem Prozess schließlich durchschlagendes Gewicht und Dauer verliehen haben, als vielmehr technische Innovationen, die besonderen Eigenschaften dieses Energieträgers sowie der Ausbau der Infrastruktur und sozio-ökonomische Liberalisierungsprozesse. ${ }^{43}$ Die von alters her bekannte, aber nie intensiv geförderte und genutzte Kohle gelangte im Zuge des Industrialisierungsprozesses in den Rang eines neuen Hauptenergieträgers. ${ }^{44}$

Der damit angedeutete ebenso expansive wie voraussetzungsvolle Transformationsprozess zum modernen Kapitalismus und zur umfassenden Kohlenutzung durchbrach die vormodernen, die agrarischen » Grenzen des Wachstums « ${ }^{45}$ Er führte zu einer gewaltigen Spirale wirtschaftlicher Leistungssteigerung und ermöglichte ein bedeutendes demographisches Wachstum. Die Wachstumsprozesse beruhten nun allerdings, ohne dass die Nutzung des Holzes auf allen Verwendungsgebieten verdrängt wurde, auf einem erschöpfbaren »fossilen Energiesystem «. Die Dramatik dieser Einsicht führte im Pionierland der »industriellen Revolution «, in England, schon im frühen 19. Jahrhundert zu Diskussionen und Parlamentskommissionen,

40 Circulare 1801, Sp. 18-19.

41 Gleitsmann 1981, S. 83 ff.

42 Gleitsmann 1982, S. 51.

43 Vgl. Grabas 1995.

44 Eine ähnlich lange »Vorgeschichte « hat übrigens das später in den Rang einer Zentralressource rückende Erdöl (vgl. dazu Suhling 2001, S. 257 ff.)

45 Holenstein et al. 2007, S. 7 ff. 
die nach Auswegen aus einer prognostizierten neuen Knappheitssituation suchten. 46

Noch ganz in der Logik der vorindustriellen, der »hölzernen Zeit « gefangen, hat es im deutschsprachigen Raum - und nicht nur dort - im Rahmen der zwischen objektiven Befunden und subjektiven Bewertungen und Interessen schwankenden frühneuzeitlichen »Holznot-Debatte « nicht an weiteren Ansätzen gefehlt, die Ressourcenknappheit abzumildern. Ansätze hoheitlicher Holzzuteilung lassen sich in der Agrargesellschaft und auch noch im Rahmen der gewinnmaximierenden Forstwirtschaft nachweisen. Neben die Ansätze zur Erhaltung einer nachhaltigen holzbasierten Gewerbestruktur durch Begrenzungen gewerblicher Aktivitäten traten holzsparende und die Brandgefahr minimierende Bauvorschriften. Gleichzeitig gab es Ratschläge zur Vertäfelung der Innenwände und zum Einbau von Doppelfenstern und Doppeltüren als Strategien der Energieeinsparung. Die Idee, für die Dichtigkeit der Gebäude zu sorgen, kleine Stuben als »Winter-Stuben «, größere als »SommerStuben « zu gebrauchen, lag nicht fern. ${ }^{47}$ Letztlich wurden bereits damals alle Lebensbereiche und Gewohnheiten auf Holzsparmöglichkeiten hin untersucht. So findet man auch Vorschläge zur Abschaffung der Maibäume, zum mehrfach benutzbaren Holzsarg, zur Ersetzung der Holzzäune durch Hecken usw. usf. ${ }^{48}$

Es konnte in einer Gesellschaft, deren ärmere Bevölkerungsschichten ständig unter der Drohung von Hunger und Kälte standen, nicht ausbleiben, dass die Art und Weise des Holzverbrauchs durch Verfeuerung besonders intensiv in den Mittelpunkt der Kritik geriet, »[...] zumahl da das Element des Feuers der geschwindeste und unbarmherzigste Verzehrer des Holtzes bleibet «. ${ }^{49}$ Aus diesen argumentativen Zusammenhängen heraus entwickelten sich weitere besondere Bestrebungen zur Förderung der Holzeinsparung und eine umfangreiche frühtechnologische Literatur zur »Holtz-Sparkunst « in Gewerbe, Handwerk und Haushalt.

\subsection{Die Holzsparkunst als Gegenstand obrigkeitlicher Privilegierungen}

Das knappe Holz als Energielieferant, als Grundlage nicht nur des Lebens und Gedeihens von Mensch und Tier, sondern auch als Voraussetzung einer Vielzahl physikalischer und chemischer Prozesse in Gewerbe, Handwerk und Haushalt, wurde nach der Auffassung der zahlreichen Holzsparautoren geradezu verschwendet. Das zu verhindern war das Ziel einer ganz besonderen Holzsparkunst. Zur »Holtz-Sparkunst « gerechnet wurden »[...] alle die Vortheile, wodurch dem übermäßigen Verthun des Brenn-Holtzes vorgebeuget, und getrachtet wird mit wenigem Holtze viel Hitze zu Wege zu bringen. An. 1557 auf dem Reichs-Tage zu Regensspurg haben Frdr. Fröhmer, Ulr. Rundmann und Conrad Zwicke von dem Kayser und Reiche ein Privilegium erhalten, eine gewisse Kunst, wodurch sie im täglichen

46 Vgl. Sieferle 1987.

47 Vgl. von Carlowitz 1713, S. 47.

48 Radkau, Schäfer 1987, S. 190 f.

49 Vgl. Lehmann 1735. Schätzungsweise wurden von 1820 bis 1850 in Deutschland noch 80 Prozent des Holzeinschlags verbrannt; vgl. Grewe 2003, S. 74, Fußnote 32. 
Brauche viel Holtz zu ersparen gemeynet, auf eine gewisse Zeit alleine zu treiben $«{ }^{50}$ Diese Lexikonnotiz eröffnet einen Blick in die Wissenschaftsgeschichte und in eine »Herzkammer " des ursprünglich-vorwissenschaftlichen und später: des naturwissenschaftlich-technologisch fundierten Fortschritts in der Ressourcennutzung.

Beachtlich an diesem Lexikoneintrag ist die Eröffnung einer zeitlichen Perspektive bis in die Mitte des 16. Jahrhunderts hinein und in die damit verbundene »Privilegierungspraxis" des Heiligen Römischen Reiches Deutscher Nation. Diese Praxis existierte auch auf der Ebene verschiedener Landesherren. Sie bezog sich zunächst auf das Montanwesen, das mit Problemen der Wasserhaltung und Verhüttung zu kämpfen hatte. Hinzu traten aber schon bald Erfinderprivilegien auf »holzsparende Technologien ", die sich auf andere Gewerbebereiche bezogen, zum Beispiel auf das Hüttenwesen, auf salinarische Verfahren, Kalk- und Ziegelbrennereien. Zwangsläufig wurden auch die häuslichen Koch- und Heizsysteme Gegenstand von energiesparenden »Inventionen « und von Anträgen an die Obrigkeiten, die durch »vleissig nachsinnen " und »tegliche Übung « entwickelten Erfindungen zu schützen. ${ }^{51}$ In der vorindustriellen Zeit, in der in unterschiedlichem Ausmaß die nachwachsende, aber knappe Zentralressource Holz einen Engpassfaktor für die »Landesentwicklung « darstellen konnte, handelte es sich bei den holzersparenden »Inventionen " sogar um den Prototyp des damals erwünschten und herbeigesehnten (vor)wissenschaftlich-technischen Fortschritts, hierin dem »Goldmachen « nicht unähnlich.

Die meist von einem Konsortium von Erfindern oder Förderern eingereichten beziehungsweise beschriebenen Erfindungen wurden von besonderen Kommissionen der Herrschaftsträger im Rahmen eines bestimmten Verfahrens begutachtet. Das Kernstück dieses Verfahrens war typischerweise die praktische "Probe « der neuerfundenen "Kunst ", um so sicherzustellen, dass es sich bei der vorgestellten »Invention " um etwas Neues und um einen wirklichen »technischen Fortschritt « handelte, dass sie auch in der Praxis funktionierte ${ }^{52}$ und um einem Missbrauch in einer Zeit der Blüte »[...] einer neuen, sehr regsamen und zudringlichen sozialen Schicht: der Spekulanten, Projektemacher, Alchimisten, Pseudoerfinder und anderer Abenteurer, welche die Höfe des absolutistischen Fürstentums in immer steigenderem Masse bevölkerten $[\ldots]$ « entgegenzuwirken. ${ }^{53}$ Die gegen eine Gebühr ausgehändigte Urkunde (meist als Privileg, Freiheit oder Befreiung bezeichnet) gewährte für ein bestimmtes Herrschaftsgebiet und eine bestimmte Anzahl von Jahren das Recht, die Erfindung selbst herzustellen und zu vertreiben oder von "Nachahmern " dieser Kunst einen bestimmten Geldbetrag verlangen zu können. Für den Fall der unbefugten Nachahmung waren Strafen vorgesehen. Die beeindruckenden Urkunden verfehlten gegebenenfalls auch auf die angerufenen Gerichte ihre Wirkung

50 Zedler 1735 b, Sp. 711, Hervorhebung im Original.

51 Vgl. mit Hinweisen auf zahlreiche Erfinderprivilegien aus dem 16. Jahrhundert: Hoffmann 1915; Müller 1939; Pohlmann 1960; umfassend: Gleitsmann 1985.

52 Vgl. Pohlmann 1960.

53 Silberstein 1961, S. 291. 
nicht. So wurde das finanzielle Eigeninteresse, der Eigennutz, eine wesentliche Triebkraft für Erfindungen, und gleichzeitig spielte das legitimierende Argument der Gemeinnützigkeit für die Privilegierungen eine wesentliche Rolle. ${ }^{54}$ Häufig wurde in dem Antrag auf Privilegierung auf die aufgewandten Mühen, den Nutzen für die Staatskasse, die Kostenreduzierung, die Verbesserung der Arbeitsbedingungen usw. usf. hingewiesen. ${ }^{55}$ Das bedeutete im Allgemeinen, dass die »Invention « zur Erleichterung oder Überwindung eines herrschaftlichen oder gesellschaftlichen Problems vermeintlich oder tatsächlich beitragen musste. Privilegiert wurden vor allem jene Erfindungen, die gewerblich, handwerklich oder hauswirtschaftlich verwertbar waren.

Die auf die Holznot bezogenen »Holtzspar-Künste « erfüllten diese Voraussetzungen spielend. ${ }^{56}$ Die sozial-, rechts- und technikgeschichtlich höchst aufschlussreichen Holzspar-Privilegien ${ }^{57}$ sind von großer Zahl und Bedeutung. Von den aus der Literatur bekannten und im 16. Jahrhundert erteilten Privilegien bezog sich rund ein Drittel auf die Holzsparkunst ${ }^{58}$, das heißt vornehmlich auf die Einsparung von Holz als Energielieferant. Offenbar sollten materielle Anreize dazu beitragen, das »Innovationstempo « der damaligen Zeit durch einen staatlich gesteuerten Prozess der Erfindungen zu erhöhen. ${ }^{59} \mathrm{Im}$ Rahmen einer zünftlerischen Gewerbeverfassung, die sich gegen den von einer Erfindung ausgehenden Wettbewerbsdruck zur Wehr setzte, florierten diese Formen der merkantilistischen Gewerbe- und Erfinderförderung vor allem dort, wo die damaligen öffentlichen Gewalten ein erhebliches Interesse hatten und wo der enge Rahmen der Handwerkerzunftregeln bereits durchbrochen war. ${ }^{60}$

Neben die Urkunden und Einlassungen im Zuge der Privilegierungsverfahren trat bald eine regelrechte Holzsparliteratur, die sich in erheblichem Umfang auf den häuslichen Bereich bezog. Es scheint allerdings so zu sein, dass während des gesamten 16. Jahrhunderts das Motiv der Wahrung der Schutzwirkung der Privilegierung verhindert hat, vor allem haushaltsbezogene Holzsparkünste in Form von allgemein zugänglichen Schriften zu publizieren. Solche Veröffentlichungen hätten leicht zur unbefugten Nachahmung der Erfindung führen können. ${ }^{61}$ Dieses Verhalten der Erfinder traf jedoch nicht für alle Gebiete der technologischen Entwicklung zu. Maschinenschriften und technische Schau- beziehungsweise Maschinenbücher waren schon zu Beginn der Frühen Neuzeit verbreitet. Sie zeichneten durchgängig

54 Vgl. dazu auch Schulze 1986.

55 Vgl. Popplow 1998.

56 Gleitsmann 1985, S. 70 f.

57 Aus rechtshistorischer Sicht ist vor allem interessant, dass sich auch auf diesem Gebiet schon früh Tendenzen der Entwicklung zur Rechtsstaatlichkeit beobachten lassen (vgl. Pohlmann 1960).

58 Gleitsmann 1985, S. $73 \mathrm{f}$.

59 Vgl. Silberstein 1961.

60 Zycha 1939, S. $210 \mathrm{f}$.

61 Gleitsmann 1985, S. $84 \mathrm{f}$.

Leviathan, 42. Jg., 3/2014 
ein faszinierendes, die Leistungskraft und die Möglichkeiten der Technik idealisierendes Bild, verunklarten aber häufig auch technische Details. Viele der Autoren waren Inhaber von Erfinderprivilegien, die auf diese Weise Werbung in eigener Sache machten. ${ }^{62}$ Dabei ist zu beachten, dass der »Buchmarkt « noch nicht weit entwickelt war. Die Publikation detaillierter Holzsparschriften, die sich auf Herd und Ofen im häuslichen Bereich bezogen, und damit die Entstehung einer ganz speziellen frühtechnologischen Literatur begann im Wesentlichen erst Jahrzehnte nach der entsprechenden Privilegierungspraxis im Kriegsjahr $1618 .{ }^{63}$

\subsection{Wärmeerzeugung und Holzverbrauch in Herd und Ofen}

$\mathrm{Zu}$ Beginn der Frühen Neuzeit hatten »Unterkünfte«, Haus und Hauswirtschaft, Herd und Ofen bereits eine lange Entwicklung zurückgelegt. Diese Entwicklung führte über das offene Feuer in der Natur zum offenen Feuer im Haus, das ebenerdig oder in einer Feuerkuhle und ohne speziellen Rauchabzug betrieben wurde. ${ }^{64}$ Später traten die teilweise Umbauung, der Rauchfang und der Schornstein hinzu. Ein Meilenstein waren die weitgehend geschlossenen, ummauerten Feuerräume und die kastenförmigen Öfen aus eisernen Kaminplatten, die dazu beitrugen, dass die Wärme im Raum besser verteilt werden konnte. Es folgten eiserne Rund- und Kugelöfen. Zentral war auch die Entwicklung von Rauchabzugsvorrichtungen, die unnötige Wärmeverluste verhinderten und den Rauch zuverlässig abführten. Diese Neuerungen setzten die Erfindung des Eisengusses voraus und wurden in Hüttenwerken und Gießereien hergestellt. ${ }^{65}$ Zahlreiche Varianten von »Heiz- und Kochgeräten " wurden in diesem Zusammenhang entwickelt. Die Nutzung der einzelnen Stufen und Moden der Entwicklung von Herd und Ofen erfolgte nicht überall im Gleichschritt. Unterschiedlich »fortschrittliche« Feuerungsarten wurden vielmehr in Abhängigkeit von regionalen Besonderheiten, von Stand und Reichtum beziehungsweise Armut und entsprechenden Wohnverhältnissen betrieben. Vor allem die archaische, ungesunde und »unvernünftige ", die »brennholzfressende « Form der Nutzung des offenen Feuers war und ist kulturell tief verankert und hält sich bis zum heutigen Tag. Mit dem Feuer hängen immer schon soziale Elemente zusammen, gesellschaftliche und religiöse Rituale, deren Bedeutung nicht unterschätzt werden darf.

62 Popplow 1996, S. 31 ff.; Popplow 1998, S. $31 \mathrm{ff}$.

63 Gleitsmann (1985, S. 84 f.) erwähnt lediglich eine vor diesem Jahr erschienene Bildflugschrift zu holzsparenden Stuben-, Back-, Koch- und Bratenöfen, die das Datum des Jahres 1563 trägt. Faber (1950, S. 40) verweist auf die Schrift: »Holzkunst «/ Ein Verzeichnuss der figuren und neuwen öfen von der Ersparung neu erfundenen Holtzkunst bei Peter Schmidt. Mühlhausen/Elsaß 1564.

64 Wenig bekannt scheinen mir die mit den archaischen Formen der Nutzung des offenen Feuers einhergehenden historischen Gesundheits- und Lebensgefahren gewesen zu sein, also Verbrennungen, Rauchvergiftungen, Verpuffungen oder dergleichen, die wohl deshalb kaum zur Sprache kamen, weil sie sich in den Hütten der analphabetischen, armen Bevölkerungsschichten abspielten.

65 Vgl. Schrader 2001, S. 9 ff.; Faber 1950. 
Von einem bereits weit entwickelten Standard ausgehend, eröffnete der Porträtmaler und vielseitige Erfinder Frantz Keßler mit einer Schrift aus dem Jahre 1618 den Reigen der haushaltsbezogenen Holzsparliteratur. ${ }^{66}$ Er zeigte bereits deutlich, mit welchen Mitteln eine »Holzersparnis « erreicht werden sollte: durch eine »optimale Größe« der Herde und Öfen, durch einen geschlossenen Feuerraum mit zweckmäßiger Luftzufuhr, gebremsten Wärmeabzug bei Nutzung der Abwärme etwa zur Trocknung verschiedener Materialien oder zur Warmhaltung von Speisen, betrieben mit optimal aufbereitetem, vor allem mit trockenem Brennmaterial. Dazu zählte bei ihm auch bereits die damals nur in geringem Umfang genutzte Kohle. Keßler betrat mit seinen in die Ofenplatte eingesetzten Kochtöpfen die Schwelle zur »energieoptimalen « Umgestaltung des gesamten Heiz- und Küchengeschehens. Damit ist bei ihm der die Geschichte der Menschheit begleitende Problemhorizont des bloßen Feuermachens und des Feuerbewahrens bereits weit überschritten.

Keßler stellte seine Überlegungen zu »Kunstöfen «, durch deren Gebrauch man jährlich und insgesamt gesehen »ein unglaubliche Summa Geltes ersparen « könne, in den Rahmen der sozialen Probleme seiner Zeit. Während der gesamten Frühen Neuzeit lebte »[...] die Mehrzahl der Menschen in Europa und auch im Gebiet des Alten Reiches am Rande eines kaum exakt definierbaren Existenzminimums oder unterhalb einer >Armutsgrenze und war stetig von Krisenerscheinungen [...] bedroht «. ${ }^{67}$ Vom späten Mittelalter an war die Armut eine Massenerscheinung "[...] und ein strukturelles Problem der europäischen Gesellschaften «. ${ }^{68}$ Vielfach verbunden war die Armut mit subsistenzwirtschaftlichen Wirtschafts- und Lebensformen, mit der Abhängigkeit vom Ertrag des Landes und damit auch von Wind und Wetter. Verknüpft man diese Erscheinungen mit ungünstigen Wohnverhältnissen und dem Klimageschehen in der Frühen Neuzeit (»Kleine Eiszeit «), so gewinnen weitere Hinweise Keßlers an Plausibilität und Gewicht. Er thematisiert ausdrücklich die Gefahr des Erfrierens in der »kalten Winterzeit « und die zahlreichen durch die Kälte bedingten Krankheiten. Der Hinweis darauf, dass mancher »ehrlicher habhaffter Haußhalter « mehr für das Holz als für das Brot zu sorgen pflegte, und die Preissteigerungen für Holz verweisen auf weitverbreitete Krisen- und Knappheitssituationen und darauf, dass sich viele Menschen schon lange vor dem großen Privatisierungs- und Kommerzialisierungsschub im Zusammenhang mit den preußischen Reformen zu Beginn des 19. Jahrhunderts und der forstwirtschaftlich und kommerziell geprägten Waldnutzung nicht mehr aufgrund bestehender und in Anspruch genommener Rechte zum Beispiel aus einer "Gemein-Waldung " mit »benötigter Holtzung « versehen konnten, ${ }^{69}$ sondern für Holz zu zahlen hatten. Keßler betonte darüber hinaus die Gemeinnützigkeit seiner Absichten und ging, den Wert seiner Holzspartechnologien hervorhebend, davon aus, dass der Holzmangel im überflüssigen Holzverbrauch seine Ursache habe.

66 Vgl. Keßler 1618.

67 Härter 2005, S. 91.

68 Ehmer 2005, S. 1.

69 Zedler 1735 a, Sp. 706. 
Auch der Inhaber von Erfinderprivilegien, Johann Cristian Lehmann, der mit seinen »Inventionen " viel Aufsehen erregte und »[...] von Kennern der Natur-Wissenschafft nicht wenigern Beyfall erhalten « hat, ${ }^{70}$ betonte den jährlich »viele Tonnen Goldes übersteigenden " Nutzen seiner Erfindungen. Sie schonten die Wälder der Landesherren und die Kasse der »treuen Unterthanen«. Er präsentierte Öfen, die das Kochen, Braten, Sieden, Trocknen und Heizen kombinierten und mehrere Stuben gleichzeitig heizen konnten. Die »Krönung « seiner Erfindungen war ein »Oeconomie-Ofen, der die Stube heitzet, drey Brandtewein-Blasen, ein Balneum Maris, eine Kuffe von 8 Ellen mit Wasser in Sudt bringet, drey Töpffe kochet, brät, glühende Eisen machet, darret und maltzet «. Damit umreißt Lehmann den Umfang der damaligen Verwendung von Herd und Ofen in Haushaltungen, die nicht nur Orte des Konsums waren, sondern die darüber hinaus noch vielfältige produzierende und verarbeitende Funktionen hatten. Es fehlt nicht an Hinweisen, dass seine »Inventionen « zur praktischen Anwendung gelangten. Zu erwähnen ist bei dieser Vielfalt hauswirtschaftlicher Feuernutzung auch noch der Energiebedarf der damals weit verbreiteten Wasch- und Futterküchen, in denen die Kleidung gesäubert und das Viehfutter durch Hitzeeinwirkung zur Fütterung vorbereitet wurde. ${ }^{71}$

Zur Beantwortung der Frage nach der praktischen Anwendung der Erkenntnisse aus den annähernd 1.000 Titeln zur Holzsparkunst ${ }^{72}$ liegen nicht viele »belastbare " Hinweise vor. Einzelne Lieferungs- und Absatzzahlen entsprechender Herde und Öfen, ${ }^{73}$ einige »Umsetzungsgeschichten « sind überliefert. Bekannt ist, dass 1763 Friedrich der Große ein Preisausschreiben über »einen Stubenofen, so am wenigsten Holz verzehret «, durchführte, aus dem 1764 Johann Paul Baumer als Sieger hervorging. Sein innovativer Sparofen wurde später zum Inbegriff des »Berliner Kachelofens ".${ }^{74} \mathrm{Ob}$ und inwieweit die angepriesenen, auf den Haushalt, eine Stadt, ein Herrschaftsgebiet berechneten und dann gewaltigen Einsparungssummen an $\mathrm{Holz}$ oder Geld tatsächlich erreicht wurden, ob die Holzsparkünste den Bestand des Waldes vermehrt oder die Ausdehnung der dringend zur Ernährung erforderlichen Wiesen-, Feld- oder Gartenflächen befördert, den Holzpreis durch Nachfragesenkung vermindert, die massiven Verarmungserscheinungen abgemildert haben, weiß man nicht und wird flächendeckend kaum mehr rekonstruierbar sein. Dass die Holzsparliteratur für den Laien unverständlich gewesen sei und dass die Fähigkeiten der Handwerker zu gering, dass die "wirklich Armen " sich die eigentlich dringliche Investition in einen Holzsparofen oder -herd nicht leisten konnten, sind begründete Annahmen. Den Menschen in " primitiven " und schadhaften Hütten wäre vor allem auch mit Baumaßnahmen bei ihren Holzsparbemühungen geholfen worden. Der verarmte Adel, der in zugigen, dickwandigen und großdimensionierten Burgen,

70 Lehmann 1735, S. 214.

71 Grewe 2004 b, S. 119.

72 Es handelt sich offenkundig um eine grobe Schätzung. Hinzuzuzählen wären Veröffentlichungen in fremder Sprache und zahllose Erörterungen in den Zeitschriften und Zeitungen der damaligen Zeit. Zu dieser Schätzzahl vgl. Gleitsmann 1984, S. 193.

73 Ebd., S. 195.

74 Radkau, Schäfer 1987, S. 193. 
Gutshäusern oder Schlössern wohnte, wäre insbesondere durch eine andere Architektur vom Holzverbrauch entlastet worden. Die Auffassung, dass im 18. Jahrhundert vor allem steigende Holzpreise manchen Haushalt ruiniert hätten, lässt sich, was das Ausmaß anbetrifft, nicht quantifizieren. Dabei bemühten sich einige Holzsparautoren durchaus, leicht bedienbare, erschwingliche und energiesparende Konstruktionen anzubieten und den Weg zur Kohlefeuerung zu ebnen. ${ }^{75}$ Die Kohlefeuerung traf jedoch zunächst noch auf große Vorbehalte gegenüber den Gerüchen, dem Rauch und dem Ruß dieser Feuerungsart. Sie verlangte eine neuartige Handhabung und meistens auch neue Öfen, Herde und Geräte. Der Rauch konnte nicht mehr zum Räuchern genutzt, die Asche nicht mehr zur Pottascheproduktion verwendet oder verkauft werden. ${ }^{76}$ Andere Autoren und Praktiker entwickelten hingegen Varianten der »Holzsparkunst « für das reiche Bürgertum oder Pracht- und Prunköfen für vermögende adelige Kreise. Sie betrieben in ihren Schriften und mitunter auch in ihren Aktivitäten eine ganz besondere Spar- und Substitutionsstrategie. Gar nicht erreicht wurde jedoch jener Teil der armen Bevölkerung, der weder eine feste Haus- noch eine Feuerstätte besaß. Diese Menschen erlebten Holzsparkünste mitunter in den disziplinierenden oder » behandelnden « Institutionen der damaligen Zeit, in den Arbeitshäusern, den Gefängnissen und Hospitälern.

Explizit oder implizit enthielt die Holzsparliteratur, mit ihren technologischen Grundeinsichten verknüpft, Ausführungen zur »Natur « von Wärme und Feuer. In diesem Zusammenhang zeigte sich eine Tendenz, nicht bei den Erscheinungen stehenzubleiben, sondern zum Wesen des Feuers und der Wärme vorzudringen. Falsche »theoretische " Einsichten konnten dabei durchaus zu brauchbaren Herd- und Ofenkonstruktionen führen. Genaue Kalibrierungen und mathematisch abgestützte wirklich optimale Heiz- und Kochgeräte waren so allerdings nicht möglich. Was das Feuer sei, das sei eine Frage, die den »Weltweisen « so viele »secula " hindurch zu schaffen gemacht habe und sie auch jetzt noch beschäftige. Viele »Weltweise « suchten den Grund der Wärme in einem »fluido subtilissimo ", das in sehr heftiger Bewegung sei und alle Körper durchdringe und sie erwärme, vermerkt Zedlers Lexikon. Feuer sei eine spezielle Materie, es gebe "Feuer-Theilgen $" .{ }^{77}$ Obwohl die »Natur " des Feuers durch Lavoisier bereits als Oxidationsprozess erkannt und analysiert war, konnte der Verfasser einer verbreiteten Holzsparschrift aus dem Jahre 1800, Johann Christian Hoffmann, auf der Basis der Teilchen- beziehungsweise Phlogiston-Theorie schlussfolgern, "[...] daß die Holzsparkunst eigentlich darin bestehe, die Wärme oder den Wärmestoff aus den Körpern, die dergleichen enthalten, auf die zweckmäßigste und vortheilhafteste Weise zu entbinden, zu entwickeln oder herauszuziehen $\left[\ldots\right.$. «. $^{78}$ Umfangreiche Erörterungen über die Wärmeleitung schließen sich an, bevor Details entsprechender Heiz- und Kochgeräte und Sparstrategien

75 Gleitsmann 1984, S. $202 \mathrm{f}$.

76 Grewe 2004 b, S. 122 ff.

77 Zedler 1735 c, Sp. 716 ff.

78 Hoffmann 1800, S. 1, im Original teilweise gesperrt. Diese oder eine ähnliche Definitionen fanden sich noch jahrzehntelang in den Allgemeinen Enzyklopädien der damaligen Zeit. 
vorgestellt werden. Ausgehend von der Meinung, dass der Holzmangel mehr durch den erhöhten Preis als durch den »wirklichen Mangel dieses Produkts « bedingt sei, stellte auch er sich auf die Seite der armen Bevölkerung.

Auf diese Weise wurde in der Holzsparliteratur der Frühen Neuzeit etwas erkennbar, was schon am Beispiel der innovationsorientierten Ausgestaltung des Erfinderschutzes, der Innovationsförderung im »Dunstkreis « der Residenzen, ${ }^{79}$ deutlich wurde: Im Rahmen dieser Literatur wurde praktisch nutzbares Wissen kommuniziert und zur Anwendung empfohlen. Es ist erkennbar, dass mit der haushaltsbezogenen Holzsparkunst vor allem ein soziales oder besser das soziale Problem der vorindustriellen Gesellschaft, die Massenarmut beziehungsweise subsistenzwirtschaftliche Dürftigkeit, durch einen technisch-ökonomischen Ansatz bewältigt werden sollte. Es sollten aber auch hauswirtschaftliche Aktivitäten unterstützt werden. Die Holzsparkünste konnten in der Frühen Neuzeit zwar noch nicht auf die Basis von unwandelbaren und mathematisierten Naturgesetzen gestellt werden, aber die Grundlagen der Entdeckung solcher Gesetze, Beobachtung und Experiment, standen erkennbar an der Wiege der Entstehung dieses Schrifttums der Frühen Neuzeit und des Erfinderschutzes. Der Weg zwischen der mehr oder weniger theoretisch anspruchsvollen »Naturlehre " und ihrer Nutzanwendung sollte verkürzt werden. Die bloße Anzahl der auf die Holzsparkunst bezogenen Quellen und die Beteiligung prominenter Persönlichkeiten, der Höfe, der Societäten und Akademien an den entsprechenden Bestrebungen ${ }^{80}$ machen den hohen Stellenwert der energetischen Optimierung der Hauswirtschaften im technischen und armenpflegerischen Denken der Frühen Neuzeit deutlich. Unerschütterlich scheint die Überzeugung der Holzsparautoren gewesen zu sein, mit ihren "Inventionen « und Vorschlägen zur Verbesserung der Gesellschaft beitragen zu können. Nicht zuletzt verschaffte diese Auffassung ihren Schriften und »Inventionen « Bedeutung und Attraktivität.

Einen wichtigen und europaweit ausstrahlenden Kumulationspunkt dieser innovatorischen Bemühungen bildeten die Aktivitäten und Schriften des vorübergehend in pfalz-bayerischen Diensten stehenden Reichsgrafen von Rumford (1753-1814). ${ }^{81}$ Rumford war nicht nur Staatsmann, Heeresreformer, Polizeireformer, "Ernährungswissenschaftler ", Stadtplaner, empirisch arbeitender Naturforscher und Ingenieur sowie Aktivist im Rahmen der europäischen Akademie- und Societätsbewegung. Er widmete sich in diesen Eigenschaften stets zugleich dem Ziel der energetischen Optimierung der Haus- und Anstaltsökonomie. Erkennbar um die Mehrung seines Ruhmes bemüht und ausdrücklich von der Basis der in hohem Ansehen stehenden Naturlehre aus, projektierte und erprobte er »ökonomische Öfen " (sogenannte Rumfordherde), spezielle Kessel, Heizungssysteme, Lampen,

79 Troitzsch 1999, S. 8.

80 Breit diskutiert wurden die heiztechnischen Innovationen Goethes (sein Ballonofen) und der Sparofen des amerikanischen Politikers, Schriftstellers und Erfinders Benjamin Franklin; vgl. Faber 1950, S. 15.

811753 in Woburn/Massaschusetts als Benjamin Thompson geboren, wurde er in Deutschland im Jahre 1792 zum Reichsgrafen von Rumford erhoben; vgl. dazu und zu seinem abenteuerlichen Lebenslauf Reidegeld 2000, S. 550 f. 
Messgeräte usw. ${ }^{82}$ In seinen Schriften verdeutlichte er stets auf umfassende Weise den Bezug seiner Aktivitäten zur Naturlehre und dokumentierte exakt die ökonomische und wärmetechnische Vorteilhaftigkeit seiner Erfindungen. Er fand vor dem Hintergrund der durch revolutionäre Vorgänge erschütterten Staaten und Gesellschaften und der finanziell ausgezehrten Anstalten der Armenpflege größte Resonanz. Die Vorteilhaftigkeit seiner Neuerungen war anhand einfacher Beispielrechnungen leicht nachvollziehbar, und mit seiner »oeconomischen Suppe « meinte er nicht nur eine brennstoffsparende und billige, sondern gleichzeitig gesunde, sättigende und wohlschmeckende Armen- und Anstaltskost gefunden zu haben. ${ }^{83}$ Vor allem auch mit seinen "Großkochstellen « und der von ihm geplanten und durchgeführten Massenspeisung fand er in diesen unruhigen und von Armut geprägten Zeiten europaweit Nachahmung und langdauernden Nachhall. ${ }^{84}$

Schon im Jahre 1800 bewunderte auch das Ehrenmitglied der Leipziger ökonomischen Sozietät Johann Christian Hoffmann Rumfords Holzsparkünste mit dem Hinweis, dieser glaube annehmen zu müssen »[...] dass in vielen Fällen von Acht Theilen Brennmaterial Sieben Theile unnütz verschwendet werden «. ${ }^{85}$ Negativer Bezugspunkt war das weit verbreitete Heizen und Kochen mit offenem Feuer. Das Backen, Braten und Kochen von Speisen sei schon lange ein Gegenstand der Forschung gewesen und habe die Aufmerksamkeit vieler wissenschaftlich gebildeter Männer in Anspruch genommen, »[...] bis es dem oben gedachten Grafen von Rumford gelungen ist durch viele Versuche zu einiger Aufklärung in der Feuerung zu gelangen ", vermerkte die Oeconomische Encyklopädie von $1832 .{ }^{86} \mathrm{Im}$ System der gesammten Armenpflege von $1846^{87}$ war zu lesen, "Armenfreunde und große Gelehrte « hätten ihre Untersuchung der »Küche der Armen zugewandt «. Auch hier fehlt es nicht an Hinweisen auf Rumford und die von ihm entwickelte Armenspeise, die Rumfordsuppe. Seine Idee der technologischen Realisierung des Holz- beziehungsweise Energiesparens bei der Beköstigung von Menschenmassen stand für eine ganze Kette von Vorschlägen und Maßnahmen in unruhigen und »teuren und knappen « Zeiten, die Ernährung aus der Sphäre des Einzelhaushalts zu lösen und noch kosten- beziehungsweise energiesparender zu einer Speisung der »ärmeren Volksklassen « durch öffentliche Back- oder Kocheinrichtungen überzugehen. Begriffe wie Suppenanstalten, Volksküchen, Almosenküchen, Armenküchen, Volksspeisehäuser standen für solche in Kriegs- und Krisenzeiten aufblühenden Aktivitäten, an deren Durchführung dann auch Frauen und Frauenvereine ihren Anteil hatten. ${ }^{88}$ Die kursächsische »Oconomie-Deputation « setzte im späten 18. und im 19. Jahrhundert immer neue Prämien für die Abschaffung der Privat- und die Anlegung von Gemein-

82 Ebd., S. 551.

83 Rumford 1803, S. 11 ff.; Thoms 2005, S. 304 f.

84 Reidegeld 2000, S. $560 \mathrm{ff}$.

85 Hoffmann 1800, S. 19.

86 Krünitz 1832, S. 461.

87 Buß, Gérando 1846, S. 305 f.

88 Reidegeld, Reubelt 1995, S. 168 ff.

Leviathan, 42. Jg., 3/2014 
debacköfen aus. In fränkischen und schwäbischen Dörfern wurden Gemeindebacköfen benutzt. ${ }^{89}$

Mit diesen Formen und Ansätzen reichte die Holzsparkunst der Frühen Neuzeit bis in das Industriezeitalter hinein. Agrargesellschaftliche Armut und subsistenzwirtschaftliche Dürftigkeit konnten von der sich ausbreitenden Industrie zunächst nicht überwunden werden. ${ }^{90}$ Mit der Frühindustrialisierung einhergehende Formen der Armut wurden unter dem Begriff des Pauperismus diskutiert. ${ }^{91}$ Sparsame Lebensführung ganz allgemein und die Holzsparkunst sowie die Holznot blieben weiterhin ein vieldiskutiertes Thema. Noch eine ganze Weile verliefen diese Diskussionen in allerdings modifizierten Bahnen. So war gegen Ende der ersten Hälfte des 19. Jahrhunderts zu lesen: »In Folge der sich mehrenden Bevölkerung, der vielen Neubauten, der Eisenbahnanlagen und der vermehrten Ausbreitung holzverbrauchender Fabriken und Gewerke hat in neuerer Zeit der Umfang der Waldungen sehr abgenommen, und es ist deshalb mit Recht um so mehr Holzmangel zu befürchten, als die Feuerungssurrogate, wie Torf, Stein- und Braunkohlen, in Deutschland nicht in der Menge gewonnen werden, um den fehlenden Bedarf an Brennholz zu ersetzen «. ${ }^{92}$ Dieser Beitrag qualifiziert den Verbrauch nicht nachhaltiger Brennstoffe als zu gering, um dann auf die bleibende Notwendigkeit »holzersparender Koch- und Heizgeräthe" hinzuweisen.

Schon gegen Ende der Frühen Neuzeit änderte sich die Zusammensetzung der Diskutanten. Wissenschaftler und Ingenieure verdrängten die zunächst noch zahlreichen Projektemacher und Laienerfinder. Die tragfähigen und grundlegenden Einsichten der Praktiker und Autoren der Holzsparbewegung gingen wie selbstverständlich in die industrielle Fertigung von Herden und Öfen ein. Der Industrialisierungsprozess, der schließlich in manchen Industriestädten zu einer Vervielfachung der Einwohnerzahlen in wenigen Jahrzehnten führte, und der damit einhergehende Massenwohnungsbau brachten die Einführung von moderneren Koch- und Heizgeräten mit sich.

\section{Umrisse und Triebkräfte der historischen »Energiewende« und des Ressourcenwachstums}

So verließen Deutschland und in einem weiteren Sinne auch Westeuropa Schritt für Schritt die agrargesellschaftliche Existenzform, die über Jahrtausende die historische Normalform der menschlichen Existenz gewesen war. ${ }^{93}$ Das "hölzerne Zeitalter " ging zu Ende. Im Zuge einer »europäischen Sonderentwicklung « wurden die Entwicklungsgrenzen, die in der vorindustriellen Gesellschaft wirksam waren, aufgehoben. Engpässe und Ressourcenknappheiten der vorindustriellen Gesellschaft wur-

89 Radkau, Schäfer 1987, S. 191.

90 Zapf 1985, S. 60.

91 Reidegeld 2006, S. 76 ff.

92 Allgemeine deutsche Real-Encyklopädie 1845, S. 269.

93 Sieferle 2003, S. 9. 
den beseitigt. Die Kohle avancierte zum Hauptenergieträger und zur Hauptquelle volkswirtschaftlicher Kraft. Sie und die nachgelagerten Eisen- und Stahlindustrien entwickelten sich bald zu bedeutenden ökonomischen Wachstumsfaktoren, ${ }^{94}$ sie führten die westeuropäischen Gesellschaften in den »Hochkapitalismus « ${ }^{95} \mathrm{Im}$ Mitund Gegeneinander der Kräfte des »Fortschritts « und der »Beharrung « erfolgte mit dem verstärkten Einsatz der Kohle, des Stadt- und Erdgases und später des Erdöls der Übergang zu einem vorwiegend »fossilen Energiesystem «. Eine »vorindustriellorganische « wurde durch eine »industriell-mineralische Ökonomie « überformt. ${ }^{96}$ Die fundamentale Bedeutung von Holz und Wald relativierte sich durch die Nutzung der Steinkohle und von anderen fossilen Energieträgern. Ausgerechnet auf ein sehr umfangreiches, mit vorindustrieller Technik nicht erreichbares, aber letztlich doch endliches Energiereservoir wurde die industrielle Gesellschaft aufgebaut. Die Ressourcenknappheit wurde so zugleich überwunden und in die fernere Zukunft vertagt. ${ }^{97}$

Im Unterschied zum Holz waren die fossilen Energieträger nicht mehr unmittelbar flächen- und naturabhängig. Zunehmend konnte auch eine begrenzte lokale, regionale und nationale Ressourcenausstattung durch in der klassischen Wirtschaftstheorie hochgepriesene und immer beeindruckender werdende Importe ergänzt werden. Es musste in solchen Fällen keine Rücksicht mehr auf das lokal oder regional vorhandene Ressourcenangebot genommen werden, wie dies noch in vorindustrieller Zeit ganz überwiegend der Fall war. Dieser "Sachzwang « zu einer lokalen, naturangepassten, autark-kreislaufwirtschaftlichen Existenzform war im agrargesellschaftlichen Kontext allerdings auch in alter Zeit für die Herrschafts- und Handelszentren teilweise außer Kraft gesetzt. Sie verfügten bereits über europäische und koloniale beziehungsweise außereuropäische wirtschaftliche Ergänzungsräume und Machtmittel, um sich von örtlichen Beschränkungen zu lösen und so die Anhäufung von Reichtum und die Ernährung einer wachsenden Bevölkerung zu ermöglichen.

Interessant ist auch das Schicksal der nach forstwirtschaftlichen Grundsätzen kultivierten und verkehrstechnisch erschlossenen Waldungen. Sie wurden von ihren staatlichen und privaten Eigentümern während der »industriellen Revolution " tatsächlich in maximalem Umfang, aber tendenziell nachhaltig genutzt. Das ist umso erstaunlicher, weil das Holz durch den Einsatz fossiler Brennstoffe lediglich ein Stück aus dem Zentrum des sozialökonomischen Geschehens weggerückt wurde und der Holzbedarf und -verbrauch durch das Bevölkerungswachstum, den Städteund Eisenbahnbau, die sonstigen ökonomischen Wachstumsprozesse und später auch die Entwicklung einer Strommasteninfrastruktur insgesamt steil nach oben ging. Dadurch wurden auf makroökonomischer Ebene manche der erhofften Effekte des individuellen Energiesparens, der holzersetzenden Innovationen und der Substitutionsprozesse »überspielt«. Seit dem Jahre 1864 weist die Statistik des

94 Kiesewetter 2001, S. 86.

95 Sieferle 2003, S. 29, in Anlehnung an Sombart 1987 [1927].

96 Sieferle 2003, S. 36.

97 Grewe 2004 b, S. 56.

Leviathan, 42. Jg., 3/2014 
Deutschen Zollvereins tendenziell stark steigende Holzimporte und über lange Zeit abnehmende Holzexporte aus. ${ }^{98}$ Das Ausland wurde auch auf diesem Gebiet zu einem Ergänzungsraum der sich in Deutschland entwickelnden industriellen Zentren. Wenn und soweit im Ausland Raubbau getrieben wurde, geschah das zu Lasten der dortigen Entwicklungsmöglichkeiten. ${ }^{99}$ In einer Epoche, in der die uralten Wege des Fernhandels zu einem schon bald globalen Strom des Güter- und Warenverkehrs ausgebaut wurden, bedienten sich die industriellen Zentren auch bei anderen Ressourcen dieser Strategien der Überwindung von Mängeln bei der Ressourcenausstattung. Dies geschah sehr früh schon bei den Erzen.

Der so erzeugte Ressourcenüberfluss sorgte, im späten 18. Jahrhundert beginnend, für den mehr als 200 Jahre andauernden Wachstums- und Industrialisierungsprozess, der perspektivisch auch die Lebenslage der Bevölkerung verbesserte und zu einer Beschleunigung des technisch-industriellen Fortschritts beitrug, der nachhaltig auf »agrarisch-hölzerner Grundlage" gar nicht möglich gewesen wäre. Dieser Entwicklungsprozess zeitigte schon gegen Mitte des 19. Jahrhunderts Umweltschäden, die die spätmittelalterlichen/frühneuzeitlichen Umweltschäden durch das vorindustrielle Gewerbe und den Bergbau weit in den Schatten stellten. Aus der »Logik « dieses Prozesses heraus begann die Suche nach immer mehr und nach weiteren Ressourcen, um Engpässe und ressourcenbedingte Wachstumshemmungen und Gewinneinbrüche auszuschließen.

Dieser Blick auf den Holzmangeldiskurs, die Ressourcengeschichte und die Ressourcenentwicklung in Deutschland, diese Beschreibung eines (west)europäischen ökonomischen Sonderwegs macht nur zu leicht vergessen, dass in anderen Weltgegenden das »hölzerne Zeitalter « für die Mehrheit der Bevölkerung noch fortdauert. Obwohl sich ab dem 19. Jahrhundert die Versuche und Ansätze mehrten, durch Reformen Anschluss an die industriell-kapitalistische Entwicklung des » Westens " zu bekommen, ${ }^{100}$ verharrten und verharren noch immer zahlreiche Regionen und Staaten, insbesondere was ihre sozialen Unterschichten betrifft, in der agrargesellschaftlichen Existenzform mit den daraus resultierenden zahlreichen Restriktionen und Ressourcenknappheiten. Darunter befinden sich zahlreiche Gebiete, die im Verlaufe der Frühen Neuzeit und ebenso in den folgenden Jahrzehnten als Kolonien ökonomische Ergänzungsräume darstellten und zum europäischen Lebenskomfort beigetragen haben. Dort sind Rohstoff- und Energienot beziehungsweise die Holznot und Holzverschwendung für viele Menschen immer noch große Themen. Auf diese Zustände beziehen sich auch heute Aktivitäten zur Durchsetzung von Erkenntnissen einer modernen Holzsparkunst im Lebensalltag einer ausgedehnten Armutsbevölkerung. Die Parallelen und die Anklänge dieser Maßnahmen an die historische Holzspardiskussion und an die in der Frühen Neuzeit in Europa erprobten Holzsparkünste sind erhellend. ${ }^{101}$

98 Grewe 2003, S. 76.

99 Grewe 2004 b, S. 57.

100 Dies trifft erst recht auf das späte 20. und das 21. Jahrhundert zu, auf die Entwicklung nach dem Zusammenbruch des »kommunistischen Weltsystems «.

101 Vgl. den Beitrag von Pierre-Christian Fink in Die Zeit vom 4. Juli 2013, S. 27. 
Als bemerkenswert bleibt festzuhalten: Die hier beschriebene "Wende « der Ressourcennutzung im Übergang von der agrarischen zur industriellen Gesellschaft hat eher den Charakter einer Vermehrung der Rohstoffe und Energieträger und einer Umgewichtung der Bestandteile des »Energiemixes". Dadurch, dass aus einer behaupteten oder tatsächlichen Knappheitssituation heraus in den Industrieländern neue Rohstoffe und Energiequellen gefunden oder durch den weltweit ausgreifenden Handel verfügbar gemacht wurden, verbreitete sich in weiten Kreisen eine gewisse Sorglosigkeit und die Perspektive eines scheinbar gesicherten und grenzenlosen materiellen Fortschritts. Für informierte Kreise und erst recht rückblickend begann am Ende der Agrargesellschaft historisch gesehen »[...] ein Vabanque-Spiel, eine komplexe industrielle Struktur mit hoher Bevölkerungsdichte auf eine Zentralressource zu gründen, von deren Erschöpfbarkeit man wußte «. ${ }^{102}$ Die sich herausbildende gewinngetriebene Industriegesellschaft verließ den Pfad der Nachhaltigkeit in besonders markanter Form. Dieses »Vabanque-Spiel « wurde in einer sich wirtschaftlich liberalisierenden Welt »naturwüchsig " als Ergebnis des Wirkens politischer und gesellschaftlicher Interessen, technologischer Entwicklung und sonstiger Voraussetzungen »in Szene" gesetzt.

Der an die Bedingungen der vorindustriellen Zeit gebundene Diskurs um die Ressourcenknappheit in Form der Holznot wurde von einer durchaus auch taktischinteressenpolitisch instrumentalisierten Angst vor der Zerstörung der agrarischen Lebensgrundlagen ebenso getragen wie von der Zuversicht, die Wald- und Holzproblematik mit Mitteln des Sparens, der forstwirtschaftlichen Angebotserhöhung, der Substitution, der naturwissenschaftlich fundierten Technik und der vormodernen Staatlichkeit in den Griff zu bekommen. Es zeigten sich Ansätze und Formen, die notwendigerweise begrenzten und erneuerbaren Rohstoffe und Energieträger zu bewirtschaften und die Lebensgrundlagen einer agrarischen Gesellschaft zu erhalten. Die in der Wirtschafts- und Technikgeschichte zu beobachtende Tradition des Energie- und Rohstoffsparens zeigt zudem, dass eine höhere Rohstoff- und Energieeffizienz in Haushalt und Gewerbe einen durch den wirtschaftlichen Wachstumsprozess steil ansteigenden Gesamtenergieverbrauch nicht verhindern kann.

Der »erstbeste « Ausweg aus den engen Zwängen der agrargesellschaftlichen Epoche wurde »beherzt " und mit einer aus der älteren Geschichte bekannten Rücksichtslosigkeit beschritten. Im Schein der wirtschaftlichen Freiheitsformel und der davon angeleiteten Staats- und Wirtschaftspraxis wurden die Wachstumsgrenzen und -hemmnisse der vorindustriellen Gesellschaft beiseitegeräumt. Letztlich war das ein Weg, an dessen Ende zweifellos auch erhebliche materielle Wohlstandsgewinne der »Massen « standen und stehen. Eingetrübt wurde diese Bilanz allerdings durch einen erheblichen Ressourcenverzehr und Umweltschädigungen großen Ausmaßes, woraus die erst spät bedeutsam werdende Einsicht entstand, dass ein solch energieund konsumintensives Leben von Voraussetzungen abhängig ist, die langfristig und dauerhaft auf der begrenzten Erde nicht gewährleistet werden können. Eben dies war eine Botschaft des "Club of Rome " aus dem Jahre 1972 für eine damals wie heute wachstumsfixierte Zeit.

102 Sieferle 1982, S. 239.

Leviathan, 42. Jg., 3/2014 


\section{Resümee und Vergleich}

Für die Frühe Neuzeit lässt sich ein hinreichend dokumentierter und nicht nur auf den deutschen Sprachraum beschränkter Diskurs um Umweltzerstörung und Ressourcenknappheit nachweisen. Umfassend wurden Wege aus der Ressourcenknappheit einer ständisch geprägten Agrargesellschaft erörtert. Auch Maßnahmen zur Anpassung des Lebens und Wirtschaftens an die damals nur begrenzt zur Verfügung stehenden und nutzbaren »Schätze der Natur « wurden praktiziert. Vor allem mit der strategischen Nutzung der Argumente, der interessengebundenen Einfärbung des Diskurses, den insgesamt unzulänglichen vormodern-staatlichen Reaktionen, den technologischen Entwicklungen auf dem Gebiet der Holzsparkunst und der Diskussion und der Praxis einer nachhaltigen Forstwirtschaft lässt sich diese Zeit als »Urbild « heutiger Auseinandersetzungen um Ressourcenknappheit, Energie, Nachhaltigkeit und Zukunftsfähigkeit begreifen. Die historischen Auseinandersetzungen, Auffassungen und Maßnahmen sind insofern von ungebrochener Aktualität. Sie entbrannten damals durchgängig an Praktiken der Ressourcennutzung, die zu sozialen und politischen Problemen beitrugen, die Interessen der Mächtigen verletzten oder nach der Auffassung der damaligen Zeit das Prinzip der Nachhaltigkeit im Sinne einer fortwährenden optimalen Nutzung der Waldressourcen der Agrargesellschaft zu verletzen drohten. In der frühneuzeitlichen Gesellschaft, die den »fossilen Schatz « nicht umfassend nutzen konnte, wurden die natürlichen Grenzen und Grundlagen des Wirtschaftens und Lebens sogar stärker und drastischer erlebt als in vielen Jahrzehnten der industriell-kapitalistischen Entwicklungsepoche auf der Basis von reichlich vorhandener Kohle und Stahl.

Der Übergang von der Agrargesellschaft zur industriell-kapitalistischen Entwicklungsepoche war ein beispielloser Vorgang der »Befreiung « der ökonomischen Prozesse von feudalen, lokalen und regionalen Bindungen, von technologisch-wissenschaftlichen Begrenzungen, von stofflichen Engpässen und von sozialen, juristischen und politischen Fesseln. Es kam zu immer neuen Schüben der Mobilisierung von Ressourcen durch verbesserte Ausbeutungsmöglichkeiten der Bodenschätze, durch verbesserte Transportwege, durch die Entgrenzung von Räumen, durch die Herstellung des modernen Eigentums, durch die Entfesselung des Eigennutzes, durch gewinngetriebene Vermarktungsprozesse. Dieser so nur angedeutete Prozess der Destrukturierung überkommener und Strukturierung moderner gesellschaftlicher Verhältnisse war der Hintergrund eines beispiellosen Wachstums, aber auch neuer systemtypischer ökonomischer Krisen.

Blickt man von hier auf die jüngste Entwicklungsgeschichte westeuropäischer Nachkriegsstaaten, so hat der auf Wachstum beruhende "Fortschritt « gravierende ökologische Schattenseiten. Das "Antlitz der Erde « hat sich durch wuchernde Spuren der Ausbeutung der Bodenschätze, durch Risiken und Schadstoffemissionen, durch riesige »Ballungsräume «, durch die Beeinträchtigung der »Kulturlandschaften « und durch gewaltige »Infrastrukturen « tiefgreifend verändert. Insbesondere aber sind heute die unumkehrbaren Ressourcenumwandlungen beziehungsweise -verluste weltweit gewaltig. Eine Entwicklungsperspektive mit einem vielhundertfach gesteigerten Ressourceneinsatz wie im Rahmen der gesamten industriell-kapi- 
talistischen Entwicklung Deutschlands und Westeuropas scheint vor allem im globalen Maßstab für die Zukunft unwiederholbar zu sein. Eine solche Perspektive dürfte in den Industrieländern vermutlich politisch und sozial gar nicht mehr durchsetzbar sein. Eine durchgehende Fixierung auf das herkömmliche Fortschritts- und Wachstumsparadigma ohne Strategien des »Maßhaltens «, eine weltweite Verbreitung der westeuropäischen Wirtschafts- und Lebensweise wird in der derzeitigen Diskussion von vielen als unverantwortlich und mittelfristig als sicherer Weg in die Katastrophe angesehen. Kurzfristig können auf diesem Weg aber offensichtlich auch viele Menschen aus materieller Armut »befreit « werden.

Die unweigerlich näher rückenden Naturgrenzen der industriell-kapitalistischen Welt werden im globalen Ausmaß nur noch schwer verrückbar beziehungsweise nicht mehr übersteigbar sein. Das Wachstumsprojekt mit seinen immer gewaltiger werdenden Ressourceneinsätzen hat zu einer nicht nachhaltigen Spirale der Entdeckung und des Verbrauchs von Ressourcen geführt. Im Zuge dieser Entwicklung hat sich lediglich ein wichtiges Potenzial seit dem Ende der Frühen Neuzeit gewaltig entwickelt, die Fähigkeit zu naturwissenschaftlich-technischen Innovationen. Hieraus wurden in der neuesten Zeit Strategien entwickelt, durch ambitionierte technologische Innovationen so etwas wie Nachhaltigkeit wiederherzustellen. Nach der energietechnologischen Missgeburt der zivilen Nutzung der Atomkraft scheint sich mit den »erneuerbaren Energien « eine zukunftsfähige, nachhaltige und weniger umweltschädliche Lösung zumindest der Energieversorgung anzubahnen. Eingebunden in das durch weitere Entgrenzungen, Liberalisierungsprojekte und andere Strategien der Wachstumspolitik vorangebrachte weltökonomische Expansionsprojekt, kann sich der "Ausbau der erneuerbaren Energien « aber auch als Strategie entpuppen, alle verfügbaren Quellen nutzend, den Weg des materiellen Wachstums, des Raubbaus an der Natur und der Schädigung der Umwelt noch einmal um viele Jahrzehnte zu verlängern. In dieses Bild fügt sich ein ökologischer Diskurs, der nachgeschaltete technologische Lösungen für die Umweltschäden vorschlägt oder vom Aufbau "grüner " Industriekomplexe oder einem "nachhaltigen Wachstum " träumt und dabei die Problematik fortgesetzten materiellen »Fortschritts n nicht sieht. ${ }^{103}$

Die mittelfristig notwendige und unumgehbare Debatte um eine wahrhaft große »sozialökonomische Transformation « muss mehr umfassen als ein Umsteuern hin zu erneuerbaren Energien, zu einer Weltgesellschaft ohne Nutzung fossiler Brennstoffe. Solche Visionen, die meistens mit dem Blick auf die Klimafolgen einer »fossilistischen « Gesellschaft formuliert werden, greifen zu kurz. Der Blick in die Geschichte der Holzsparkunst und Forstwirtschaft sensibilisiert für die »Problematik « einer generellen Ressourcenknappheit, die unweigerlich auftauchen wird, wenn der Entwicklungspfad des materiellen Größenwachstums wirtschaftlicher Aktivitäten mit oder ohne erneuerbare Energien auf lange Dauer aufrechterhalten bleibt. Gerade aus der historischen Perspektive heraus erhebt sich die Frage nach der Entwicklung zu einer neuen »Normalform « der Gesellschaft, zu einer Form des Lebens und Wirtschaftens, die einen neuen Umgang mit der Natur gelernt haben muss, um

103 Vgl. Krüger 2013.

Leviathan, 42. Jg., 3/2014 
noch Jahrhunderte und Jahrtausende einen angemessenen Fortgang der Kultur- und Menschheitsgeschichte ermöglichen zu können. Schließt man naturwissenschaftlich-technische »Wunder « aus, so wird dies eine Zukunft sein, in der ein gravierender Ressourcenmangel auch in den heute hochindustrialisierten Ländern wieder eine dominierende Rolle spielen wird. Ob und wie dann erneut Strukturmerkmale und Entwicklungen aus der agrargesellschaftlichen Epoche in veränderter Form in den Vordergrund treten werden, muss hier dahingestellt bleiben. Aber die alten Formen des Umgangs mit Ressourcenknappheit sind ebenso bekannt wie die auf Dauer nicht tragfähigen Strategien, sich daraus zu befreien. Den genauen Weg in die Zukunft kann eine historische Analyse nur ein wenig erhellen. Sie kann ihn aber aufklärend, sensibilisierend, an bestimmte Wege und Irrwege erinnernd begleiten. Die Geschichte beinhaltet insoweit Erfahrungen, aus denen man lernen kann. Sie enthält zudem eine beunruhigende Perspektive.

In einer solchen Zukunft wird man dann vermutlich auch die »Helden « der von Westeuropa ausgehenden expansiven Wirtschafts- und Lebensweise in ihrer ganzen Ambivalenz erkennen. Es wird schmerzhaft deutlich werden, dass sie bewusst oder unbewusst, durch ihre Aktivitäten und Schriften selbst oder durch die Instrumentalisierung ihrer »Lebensleistungen « zum »Vabanque-Spiel « einer langfristig nicht zukunftsfähigen und letztlich ruinösen Wirtschafts- und Lebensform beigetragen haben.

\section{Literatur}

Abelshauser, Werner 1994. "Einleitung «, in Umweltgeschichte, hrsg. v. Abelshauser, Werner, S. 7-10. Göttingen: Vandenhoek \& Ruprecht.

Allgemeine deutsche Real-Encyklopädie 1845. »Holz«, in Allgemeine deutsche Real-Encyklopädie für die gebildeten Stände. Conversations-Lexikon. 9. Originalauflage, S. 269. Leipzig: F. A. Brockhaus.

Bader, Karl S.; Dilcher, Gerhard 1999. Deutsche Rechtsgeschichte. Berlin, Heidelberg, New York: Springer.

Bartels, Christoph 2000. »Mittelalterlicher und frühneuzeitlicher Bergbau im Harz und seine Einflüsse auf die Umwelt ", in Mensch - Natur - Technik, hrsg. v. Hauptmeyer, Carl-Hans, S. 31-51. Bielefeld: Verlag für Regionalgeschichte.

Beck, Rainer 2003. Ebersberg oder das Ende der Wildnis. München: C. H. Beck.

Brüggemeier, Franz-Josef 2003. »Umweltgeschichte - Erfahrungen, Ergebnisse, Erwartungen «, in Archiv für Sozialgeschichte 43, S. 1-18.

Buß, Franz Joseph von; Gérando, Joseph-Marie de 1846. System der gesammten Armenpflege, 1846. Nach den Werken des Herrn von Gérando und nach eigenen Ansichten von Dr. Franz Joseph von Buß. Dritter Band. Stuttgart: Verlag der Steinkopf'schen Buchhandlung.

Carlowitz, Hannß Carl von 1713. Sylvicultura oeconomica Oder Haußwirthschaftliche Nachricht und Naturgemäße Anweisung Zur Wilden Baum-Zucht. Leipzig: Johann Friedrich Braun.

Circulare 1801. »Circulare an sämmtliche Regierungen und Consistoria, daß die Kirchen- und Schul-Bedienten auf ihren dazu schicklichen Dienst-Ländereyen weiche Holzarten anzuziehen angewiesen werden sollen. De Dato Berlin, den 8ten Januar 1801 ", in Novum corpus constitutionum Prussico-Brandenburgensium praecipue Marchicarum, Band XI, Sp. 17-18.

Dietz, Burkhard 1997. »Wirtschaftliches Wachstum und Holzmangel im bergisch-märkischen Gewerberaum vor der Industrialisierung ", in Jahrbuch des Vereins für Orts- und Heimatkunde in der Grafschaft Mark 97, S. 143-183.

Ehmer, Josef 2005. »Vorwort ", in Aktuelle Tendenzen der historischen Armutsforschung, hrsg. v. Kühberger, Christoph; Sedmak, Clemens, S. 1-2. Wien: LIT Verlag. 
Ernst, Christoph 2000. »Forstgesetze in der Frühen Neuzeit «, in Policey und frühneuzeitliche Gesellschaft, hrsg v. Härter, Karl, S. 341-381. Frankfurt a. M.: Vittorio Klostermann.

Faber, Alfred 1950. 1000 Jahre Werdegang von Herd und Ofen. München: Oldenbourg.

Fink, Pierre-Christian 2013. »Öfen für alle. Die Hälfte der Menschheit kocht noch über offenem Feuer. Das will die andere Hälfte jetzt ändern ", in Die Zeit vom 4. Juli 2013, S. 27.

Freytag, Niels 2006. »Deutsche Umweltgeschichte - Umweltgeschichte in Deutschland «, in Historische Zeitschrift 283, 2, S. 383-407.

Gleitsmann, Rolf-Jürgen 1981. "Aspekte der Ressourcenproblematik in historischer Sicht ", in Scripta Mercaturae 15, 2, S. 33-89.

Gleitsmann, Rolf-Jürgen 1982. »Die Haubergwirtschaft des Siegerlandes als Beispiel für ressourcenschonende Kreislaufwirtschaft", in Scripta Mercaturae 16, 1, S. 21-54.

Gleitsmann, Rolf-Jürgen 1984. »Energiesparende Technologie um 1800 «, in Der Märker 33, 5, S. 191-203.

Gleitsmann, Rolf-Jürgen 1985. » Wir wissen aber, Gott lob, was wir thuen und technologischer Wandel im 16. Jahrhundert ", in Zeitschrift für Unternehmensgeschichte 30, S. 69-95.

Gleitsmann, Rolf-Jürgen 1989. »Und immer wieder starben die Wälder: Ökosystem Wald, Waldnutzung und Energiewirtschaft in der Geschichte", in Mensch und Umwelt in der Geschichte, hrsg. v. Calließ, Jörg; Rüsen, Jörn; Striegnitz, Meinfried, S. 175-204. Pfaffenweiler: Centaurus.

Grabas, Margrit 1995. »Krisenbewältigung oder Modernisierungsblockade? «, in Jahrbuch für europäische Verwaltungsgeschichte 7, S. 43-75.

Grewe, Bern-Stefan 2003. "Das Ende der Nachhaltigkeit? « in Archiv für Sozialgeschichte 43, S. 61-79.

Grewe, Bernd-Stefan 2004 a. » >Man sollte sehen und weinen!< Holznotalarm und Waldzerstörung vor der Industrialisierung ", in Wird Kassandra heiser? Die Geschichte falscher Ökoalarme, hrsg. v. Uekötter, Frank; Hohensee, Jens, S. 24-41. Stuttgart: Franz Steiner.

Grewe, Bernd-Stefan 2004 b. Der versperrte Wald. Ressourcenmangel in der bayerischen Pfalz (1814-1870). Köln, Weimar, Wien: Böhlau.

Grober, Ulrich 2001. »Die Idee der Nachhaltigkeit als zivilisatorischer Entwurf «, in Aus Politik und Zeitgeschichte B 24, S. 3-5.

Härter, Karl 2005. »Recht und Armut", in Aktuelle Tendenzen der historischen Armutsforschung, hrsg. v. Kühberger, Christoph; Sedmak, Clemens, S. 91-125. Wien: LIT Verlag.

Hasel, Karl 1974. Zur Geschichte der Forstgesetzgebung in Preußen. Frankfurt a. M.: J. D. Sauerländers Verlag.

Hempel, Brigitte 1980. Der Entwurf einer Polizeiordnung für das Herzogtum Sachsen-Lauenburg aus dem Jahre 1591. Frankfurt a. M., Bern, Cirencester: Peter D. Lang.

Hölzl, Richard 2010. Umkämpfte Wälder. Frankfurt a. M., New York: Campus.

Hoffmann, Fritz 1915. »Beiträge zur Geschichte des Erfindungsschutzes in Deutschland im sechzehnten Jahrhundert ", in Zeitschrift für Industrierecht 10, 8, S. 85-93; 10, 9, S. 97-105; 10, 10, S. 109-118.

Hoffmann, M. Johann Christian 1800. Holz-Spar-Kunst. Ein Buch für Jedermann. Erster Theil. Leipzig: Selbstverlag (Zweiter Theil: 1804).

Holenstein, André; Stuber, Martin; Gerber-Visser, Gerrendina 2007. "Nützliche Wissenschaft und Ökonomie im Ancien Régime", in Nützliche Wissenschaft und Ökonomie im Ancien Régime. Cardanus-Jahrbuch für Wissenschaftsgeschichte, Band 7, hrsg. v. Holenstein, André; Stuber, Martin; Gerber-Visser, Gerrendina, S. 7-16. Heidelberg: Palatina.

Karafyllis, Nicole C. 2002. " Nur soviel Holz einschlagen, wie nachwächst $•$ Die Nachhaltigkeitsidee und das Gesicht des deutschen Waldes im Wechselspiel zwischen Forstwissenschaft und Nationalökonomie", in Technikgeschichte 69, 4, S. 247-273.

Keßler, Frantz 1618. Holtzsparkunst. Oppenheim: Johan Theodori de Bry.

Kiesewetter, Hubert 2001. »Regionale Steinkohlenvorkommen und Industrialisierung in Europa «, in Scripta Mercaturae 35, 2, S. 65-90.

Koselleck, Reinhart 1989. Preußen zwischen Reform und Revolution. München: Deutscher Taschenbuch Verlag.

Krüger, Timmo 2013. "Das Hegemonieprojekt der ökologischen Modernisierung «, in Leviathan 41,3, S. 422-455.

Leviathan, 42. Jg., 3/2014 
Krünitz, Johann Georg 1832. »Sparsiedeherd «, in Oeconomische Encyklopädie, Band 156, hrsg. v. Krünitz, Johann Georg, S. 460-488. Berlin: Pauli.

Lehmann, D. Johann Cristian 1735. Utilitatis Physicae verae Specim.(ine) VI. S. Artis lucrandi lignum, d.i. Der Holtz-Spahr-Kunst Erste Continuation. Selbstverlag.

Lohrmann, Dietrich 1979. »Energieprobleme im Mittelalter: Zur Verknappung von Wasserkraft und Holz in Westeuropa bis zum Ende des 12. Jahrhunderts ", in Vierteljahrschrift für Sozialund Wirtschaftsgeschichte 66, 3, S. 297-316.

Meadows, Dennis L. et al. 1972. Die Grenzen des Wachstums. Bericht des Club of Rome zur Lage der Menschheit. Stuttgart: Deutsche Verlags-Anstalt.

Meyer, Christian Friedrich 1851. Der frühere und dermalige Stand der staatswirthschaftlichen, forstlichen und rechtlichen Verhältnisse bei den Waldungen und Jagden in Deutschland. Nürnberg: Verlag der Riegel und Wießner'schen Buchhandlung.

Mooser, Josef, 1984. »>Furcht bewahrt das Holz<. Holzdiebstahl und sozialer Konflikt in der ländlichen Gesellschaft 1800-1850 an westfälischen Beispielen ", in Räuber, Volk und Obrigkeit, hrsg. v. Reif, Heinz, S. 43-99. Frankfurt a. M.: Suhrkamp.

Müller, Hans 1939. »Patentschutz im deutschen Mittelalter ", in Gewerblicher Rechtsschutz und Urheberrecht 44, 12, S. 936-953.

Pohlmann, Hansjörg 1960. »Neue Materialien zur Frühentwicklung des deutschen Erfinderschutzes im 16. Jahrhundert ", in Gewerblicher Rechtsschutz und Urheberrecht 62, 6, S. 272-283.

Popplow, Marcus 1996. »Erfindungsschutz und Maschinenbücher: Etappen der Institutionalisierung technischen Wandels in der Frühen Neuzeit ", in Technikgeschichte 63, 1, S. 21-46.

Popplow, Marcus 1998. Neu, nützlich und erfindungsreich. Die Idealisierung von Technik in der Frühen Neuzeit. Münster et al.: Waxmann.

Prass, Reiner 1996. »Verbotenes Weiden und Holzdiebstahl «, in Archiv für Sozialgeschichte 36, S. 51-68.

Radkau, Joachim 1986. "Warum wurde die Gefährdung der Natur durch den Menschen nicht rechtzeitig erkannt? ", in Ökologische Probleme im kulturellen Wandel, hrsg. v. Lübbe, Hermann; Ströker, Elisabeth, S. 47-78. München, Paderborn: Wilhelm Fink/Ferdinand Schöningh.

Radkau, Joachim 1999. "Das 'hölzerne Zeitalter« und der deutsche Sonderweg in der Forsttechnik «, in »Nützliche Künste". Kultur- und Sozialgeschichte der Technik im 18. Jahrhundert, hrsg. v. Troitsch, Ulrich, S.97-117. Münster et al.: Waxmann.

Radkau, Joachim; Schäfer, Ingrid 1987. Holz. Ein Naturstoff in der Technikgeschichte. Reinbek bei Hamburg: Rowohlt.

Reidegeld, Eckart 2000. »Naturlehre, Ökonomie und Armenpflege - Vom Wesen und von der Bedeutung einer sozialreformerischen Innovation ", in Zeitschrift für Sozialreform 46, 6, S. 548-566.

Reidegeld, Eckart 2006. Staatliche Sozialpolitik in Deutschland. Band I. 2., überarbeitete und erweiterte Auflage. Wiesbaden: VS Verlag für Sozialwissenschaften.

Reidegeld, Eckart; Reubelt, Beatrice 1995. "Die Mahlzeitennothilfe in Deutschland ", in neue praxis 2, S. 167-182.

Reith, Reinhold 2011. Umweltgeschichte der Frühen Neuzeit. München: Oldenbourg.

Rumford, Benjamin Graf von 1803. Kleine Schriften politischen, ökonomischen und philosophischen Inhalts. Nach der zweyten vermehrten Ausgabe aus dem Englischen übersetzt. Dritter Band. Weimar: Verlag des Landes-Industrie-Comptoirs.

Schäfer, Ingrid 1992. Ein Gespenst geht um. Politik mit der Holznot in Lippe 1750-1850. Detmold: Selbstverlag des Naturwissenschaftlichen und Historischen Vereins für das Land Lippe e. V.

Schrader, Mila 2001. Gusseisenöfen und Küchenherde. Suderburg-Hösseringen: Edition :Anderweit.

Schulze, Winfried 1986. "Vom Gemeinnutz zum Eigennutz«, in Historische Zeitschrift 243, S. 591-626.

Sieferle, Rolf Peter 1982. Der unterirdische Wald. München: C. H. Beck.

Sieferle, Rolf Peter 1987. "Energie «, in Besiegte Natur, hrsg. v. Brüggemeier, Franz-Josef; Rommelspacher, Thomas, S. 20-41. München: C. H. Beck. 
Sieferle, Rolf Peter 2003. Der europäische Sonderweg: Ursachen und Faktoren. 2., erweiterte Auflage. Stuttgart: Breuninger Stiftung.

Silberstein, Marcel 1961. Erfindungsschutz und merkantilistische Gewerbeprivilegien. Zürich: Polygraphischer Verlag.

Sombart, Werner 1987 [1916]. Der moderne Kapitalismus. Zweiter Band, zweiter Halbband. München: Deutscher Taschenbuch Verlag.

Sombart, Werner 1987 [1927]. Der moderne Kapitalismus. Dritter Band, erster Halbband. München: Deutscher Taschenbuch Verlag.

Strittmatter, Werner 1986. "Wurde die Gefährdung der Natur durch den Menschen nicht rechtzeitig erkannt? Zur Diskussion der Thesen Radkaus «, in Ökologische Probleme im kulturellen Wandel, hrsg. v. Lübbe, Hermann; Ströker, Elisabeth, S. 79-92. München, Paderborn: Wilhelm Fink/Ferdinand Schöningh.

Suhling, Lothar 2001. »Erdöl und Erdölprodukte", in Europäische Technik im Mittelalter 800 bis 1400. Tradition und Innovation. Ein Handbuch, hrsg. v. Lindgren, Uta, S. 257-264. Berlin: Gebrüder Mann.

Thoms, Ulrike 2005. Anstaltskost im Rationalisierungsprozeß. Stuttgart: Franz Steiner.

Troitzsch, Ulrich 1999. "Einführung «, in »Nützliche Künste«. Kultur- und Sozialgeschichte der Technik im 18. Jahrhundert, hrsg. v. Troitzsch, Ulrich, S. 7-10. Münster et al.: Waxmann.

Volckart, Oliver 1998. »Die Wirtschaftsordnung der Ständegesellschaft « in Scripta Mercaturae 32,2, S. 44-84.

Zapf, Wolfgang 1985. »Die Wohlfahrtsentwicklung in Deutschland seit der Mitte des 19. Jahrhunderts ", in Sozialgeschichte der Bundesrepublik Deutschland, hrsg. v. Conze, Werner; Lepsius, M. Rainer, S. 46-65. Stuttgart: Klett-Cotta.

Zedler, Johann Heinrich 1735 a. »Holtz-Schlag ", in Grosses vollständiges Universal Lexicon aller Wissenschaften und Künste, Band 13, hrsg. v. Zedler, Johann Heinrich, Sp. 706-708. Leipzig, Halle: Selbstverlag.

Zedler, Johann Heinrich 1735 b. »Holtzspar-Kunst «, in Grosses vollständiges Universal Lexicon aller Wissenschaften und Künste, Band 13, hrsg. v. Zedler, Johann Heinrich, Sp. 711. Leipzig, Halle: Selbstverlag.

Zedler, Johann Heinrich 1735 c. »Feuer «, in Grosses vollständiges Universal Lexicon aller Wissenschaften und Künste, Band 9, hrsg. v. Zedler, Johann Heinrich, Sp. 716-743. Halle, Leipzig: Selbstverlag.

Zedler, Johann Heinrich 1747. "Wald «, in Grosses vollständiges Universal Lexicon aller Wissenschaften und Künste, hrsg. v. Zedler, Johann Heinrich, Sp. 1145-1194. Leipzig, Halle: Selbstverlag.

Zycha, Adolf 1939. »Beitrag zur Frühgeschichte des deutschen Erfinderrechts «, in Zeitschrift der Savigny-Stiftung für Rechtsgeschichte... Germanistische Abteilung 59, S. 208-232.

Leviathan, 42. Jg., 3/2014 
Zusammenfassung: Von dem Beginn der Frühen Neuzeit bis ins 19. Jahrhundert hinein wurde in deutschsprachigen Quellen ein intensiver Diskurs um Ressourcenknappheit geführt. Er kreiste um das Holz als dem Hauptenergiespender und dem zentralen Bau- und Werkstoff in der Agrargesellschaft. Ausgehend vom Szenario einer drohenden oder tatsächlichen Holznot und einer Waldverwüstung, wurden im historischen Kontext Wege aus der Ressourcenknappheit debattiert. Eine nachhaltige Forstwirtschaft und Projekte der Holzsparkunst wurden thematisiert. Im Rahmen der im 19. Jahrhundert sich entwickelnden industriell-kapitalistischen Wirtschaftsform verstummte diese Diskussion. Durch den Einsatz der Kohle und durch andere verbesserte Bedingungen des Wirtschaftens wurde der Weg zu einem säkularen, nicht nachhaltigen und umweltschädlichen Wachstum gebahnt. Überlegungen zur Tragfähigkeit eines solchen Weges und zur Notwendigkeit des Lebens und Produzierens innerhalb der Naturgrenzen schließen den Beitrag ab.

Stichworte: Agrargesellschaft, Diskurs, Forstwirtschaft, Holz, Holzsparkunst, Industriegesellschaft, Kapitalismus, Nachhaltigkeit, Ressourcenknappheit, Wald

\section{Sustainable forestry and fuel saving inventions - early strategies of dealing with the shortage of resources}

Summary: From the beginning of the early modern history to the first decades of the nineteenth century there was an intensive discussion about the shortage of resources in German written documents and books. This discourse was concerned with wood as the principal resource in the agrarian society. Beginning with the scenario of a wood shortage and a forthcoming or current large-scale destruction of forests ways and means were discussed to overcome the shortage of resources. The strategies to overcome the wood shortage were characterized as »Holzsparkunst «. That included measures to increase the supply of wood by means of sustainable forestry and strategies to reduce the consumption. The strategies included technological improvements of stoves for cooking and heating homes and of furnaces in manufactories in a preindustrial context. During the breakthrough to industrialization in the nineteenth century the above mentioned discourse grew silent because of the increasing use of coal and better conditions for economic activities that finished the extreme resource shortage. A secular non-sustainable growth was the result of this constellation. The necessity of a way of living and producing within the limits of nature is discussed at the end of the essay.

Keywords: agrarian society, capitalism, discourse, forest, forestry, industrial society, shortage of resources, shortage of wood, sustainability, wood

Autor

Prof. Dr. Eckart Reidegeld

Fachhochschule Dortmund

Fachbereich Angewandte Sozialwissenschaften

Postfach 105018

44047 Dortmund

reidegeld@fh-dortmund.de 